ARTICLE

\title{
Radiation-induced DNA damage and repair effects on 3D genome organization
}

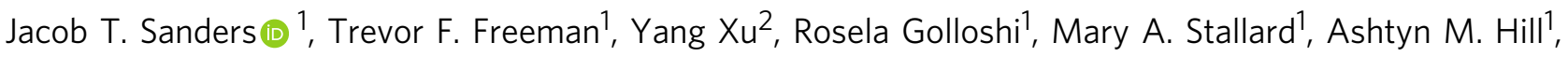 \\ Rebeca San Martin (1) ${ }^{1}$, Adayabalam S. Balajee ${ }^{3} \&$ Rachel Patton McCord (10) ${ }^{1,2}$
}

The three-dimensional structure of chromosomes plays an important role in gene expression regulation and also influences the repair of radiation-induced DNA damage. Genomic aberrations that disrupt chromosome spatial domains can lead to diseases including cancer, but how the 3D genome structure responds to DNA damage is poorly understood. Here, we investigate the impact of DNA damage response and repair on 3D genome folding using $\mathrm{Hi}-\mathrm{C}$ experiments on wild type cells and ataxia telangiectasia mutated (ATM) patient cells. We irradiate fibroblasts, lymphoblasts, and ATM-deficient fibroblasts with $5 \mathrm{~Gy} X$-rays and perform $\mathrm{Hi}-\mathrm{C}$ at 30 minutes, 24 hours, or 5 days after irradiation. We observe that 3D genome changes after irradiation are cell type-specific, with lymphoblastoid cells generally showing more contact changes than irradiated fibroblasts. However, all tested repairproficient cell types exhibit an increased segregation of topologically associating domains (TADs). This TAD boundary strengthening after irradiation is not observed in ATM deficient fibroblasts and may indicate the presence of a mechanism to protect 3D genome structure integrity during DNA damage repair.

\footnotetext{
${ }^{1}$ Department of Biochemistry \& Cellular and Molecular Biology, University of Tennessee, 309 Ken and Blaire Mossman Bldg 1311 Cumberland Ave, Knoxville, TN 37996, USA. ${ }^{2}$ UT-ORNL Graduate School of Genome Science and Technology, University of Tennessee, Knoxville, TN 37996, USA. ${ }^{3}$ Radiation Emergency Assistance Center and Training Site, Cytogenetics Biodosimetry Laboratory, Oak Ridge Institute for Science and Education, Oak Ridge Associated Universities, Oak Ridge, TN 37830, USA. ${ }^{凶}$ email: rmccord@utk.edu
} 
onizing radiation (IR) is a well-known carcinogen, and IR exposure inflicts a wide spectrum of DNA lesions among which the double strand break (DSB) is the most lethal lesion. Mis-rejoining of DSBs leads to chromosome translocations, some of which may be oncogenic in nature. Therefore, efficient DNA repair is necessary to maintain genome integrity by suppressing chromosome translocations and genomic alterations ${ }^{1}$. Most studies thus far have focused on IR-induced DNA damage and repair at the level of the linear DNA sequence with a special emphasis on mutations, cancer induction, and downstream effects for cell death and organ/tissue damage. But the human genome is not only a linear DNA sequence, but is also organized in a three-dimensional (3D) structure, which influences the proper regulation of transcription, replication, and repair ${ }^{2-5}$. Considering the importance of $3 \mathrm{D}$ genome structure in various cellular activities, it is important to understand whether IRinduced DNA damage alters the genome architecture and whether the 3D genome architecture is restored after DNA repair.

Translocations, deletions, and other genomic aberrations that may follow DNA damage can lead to cancer by directly mutating genes or altering their regulation ${ }^{6,7}$. Recently, it has become clear that the disruption of $3 \mathrm{D}$ genome domains can also be oncogenic $^{8}$. Does the process of DNA repair protect the 3D folding of the genome as well as the linear DNA sequence? Certain cell types are considered to be more radiosensitive than others, but little is known about what contributes to their radiosensitivity. It has been previously demonstrated that chromosome folding states and chromosome positioning can have a significant impact on which translocations occur ${ }^{7,9}$. Such initial 3D genome factors and local chromatin states may also influence DNA repair after $\mathrm{IR}^{10,11}$. Thus, it is possible that pre-existing $3 \mathrm{D}$ genome structure states help to explain why certain cell types are more sensitive to radiation.

Previous studies suggest that DNA repair efficiency may differ for heterochromatin and euchromatin ${ }^{12,13}$. Heterochromatic regions may be more mobile and move to DNA repair sites, where they decondense ${ }^{14,15}$. Condensation or decondensation of specific chromatin regions may not be determined by their preexisting histone modifications ${ }^{14}$, suggesting that other factors may contribute to changes in $3 \mathrm{D}$ genome structure after DNA damage. One previous study demonstrated spatial clustering of DSBs in active genes by inducing specific breaks and measuring their interactions with Capture $\mathrm{Hi}-\mathrm{C}^{16}$. This suggests that changes in the structure of local genome domains may happen at a broader scale after IR. Additionally, CCCTC-binding factor (CTCF) and cohesin have been shown to be early responders to DNA damage induced by $\mathrm{IR}^{17-20}$. These proteins have also been recently demonstrated to play significant roles in chromosome folding ${ }^{21-24}$, contributing to the formation of topologically associating domains (TADs). These genomic domains interact more frequently within themselves than with other regions and appear to help bring genes into contact with appropriate regulatory elements 25,26 . The disruption of TAD boundaries can lead to developmental diseases and cancer ${ }^{27,28}$. The prevailing model of TAD formation proposes that CTCF helps to establish TAD boundaries by blocking the loop extruding activity of cohesin ${ }^{23}$. Rapid recruitment of CTCF and cohesin to the sites of DNA damage may indicate that preservation of $3 \mathrm{D}$ genome architecture after DNA repair completion is important for genome integrity.

The above-mentioned studies suggest that changes occur in the $3 \mathrm{D}$ genome structure at multiple levels of its hierarchical organization in response to IR. However, the few studies that have examined chromosome contact changes after DNA damage thus far have focused on changes around specific induced break sites $^{16,29}$. While this is very valuable for studying local detailed structure mechanisms, various evidence suggests that DNA damage at one location can affect chromosome structures far away from the site of damage ${ }^{11,30}$. Further, different $3 \mathrm{D}$ genome structure responses may occur when breaks happen in different genomic locations. Therefore, we seek to capture general principles of how widespread and random IR-induced damage and the subsequent repair of DNA influences genome organization across length scales in a cell-type-specific manner. To accomplish this, we employ chromosome conformation capture followed by high throughput sequencing (Hi-C) to characterize genome organization across a population of cells before and after exposure to $\mathrm{X}$-rays $(5 \mathrm{~Gy})^{31}$. In a previous study, we used multi-color fluorescence in situ hybridization (mFISH) and low-resolution $\mathrm{Hi}$ $\mathrm{C}$ data on human blood cells and lymphoblasts to examine changes in the contacts and positioning of whole chromosomes at $24 \mathrm{~h}$ post-irradiation. We found that while individual cells harbored a variety of chromosomal translocations as observed by $\mathrm{mFISH}$, at a population level, no recurrent translocations were evident in the $\mathrm{Hi}-\mathrm{C}$ data ${ }^{6}$. Here, with additional cell types and repair mutants at more timepoints and higher resolution, we assay the cell-type-specific effects of IR on the hierarchical layers of genome organization. We perform $\mathrm{Hi}-\mathrm{C}$ on fibroblasts and lymphoblastoid cells at $30 \mathrm{~min}$ and $24 \mathrm{~h}$ after exposure to $5 \mathrm{~Gy}$ $\mathrm{X}$-rays. Changes in the overall genome organization are observed in the irradiated human fibroblasts and lymphoblastoid cells when compared to their nonirradiated counterparts. Human lymphoblastoid cells (GM12878) exhibit more evident changes in genome-wide $3 \mathrm{D}$ organization when compared to fibroblasts. However, both cell types exhibit a strengthening of TAD insulation, suggesting that TADs are more segregated after X-ray exposure. This effect persists at 5 days post-IR. To determine whether increased TAD insulation after IR is linked to the DNA damage/repair pathway, we examine the $3 \mathrm{D}$ genome organization changes in cells with compromised double strand break repair: human fibroblasts deficient in the ataxia telangiectasia mutated (ATM) gene. We observe that the TAD strengthening that occurs in several repair-proficient cell types after IR does not occur in ATM-deficient cells, suggesting that this genome structure change is dependent on the ATM DNA repair pathway.

\section{Results}

Skin fibroblasts exhibit subtle changes in genome architecture after IR. To determine how X-ray irradiation affects genome organization, we first irradiated confluent BJ-5ta foreskin fibroblasts with $5 \mathrm{~Gy} \mathrm{X}$-rays and analyzed cells at $30 \mathrm{~min}$ and $24 \mathrm{~h}$ post-irradiation. The majority of cells exposed to $5 \mathrm{~Gy} \mathrm{X}$-rays show chromosomal damage and translocations ${ }^{6}$. Because cells at different cell cycle phases (G1, S, and G2/M) respond differently to IR exposure, we grew the fibroblasts to a confluent, contactinhibited state in which most cells are in G0/G1 phase (Supplementary Fig. 1a) ${ }^{32,33}$. To confirm the induction of DSBs by IR, immunofluorescence and western blot analyses were performed for phosphorylated-H2AX $(\gamma-\mathrm{H} 2 \mathrm{AX})$, a surrogate marker of $\mathrm{DSBs}^{34,35}$. We observed that X-ray irradiated BJ-5ta cells showed an elevated level of $\gamma$-H2AX protein at $30 \mathrm{~min}$ post-exposure followed by a decline at $24 \mathrm{~h}$ post-IR, indicating an efficient repair of DNA DSBs (Fig. 1a and Supplementary Fig. 2a, b). There are remaining unrepaired foci at $24 \mathrm{~h}$ after IR, some of which are slowly repaired by $96 \mathrm{~h}$ post-IR, but others of which remain unrepaired until cells are passaged and allowed to proceed through cell division (Supplementary Fig. 3).

To measure changes in genome-wide chromosome contacts after IR, Hi-C was performed immediately before IR exposure and at $30 \mathrm{~min}$ and $24 \mathrm{~h}$ after exposure (see Methods and Supplementary Table 1 for statistics, processing, and replicate 


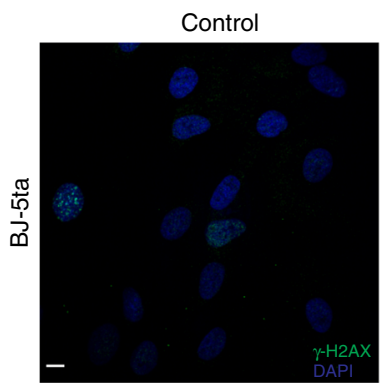

b

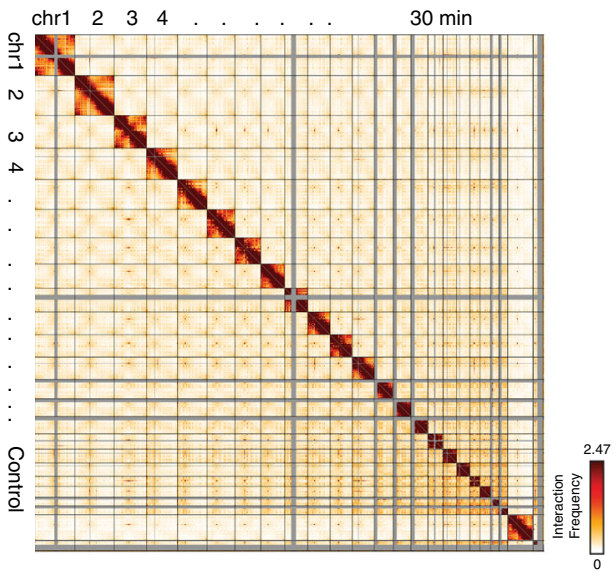

$30 \min$

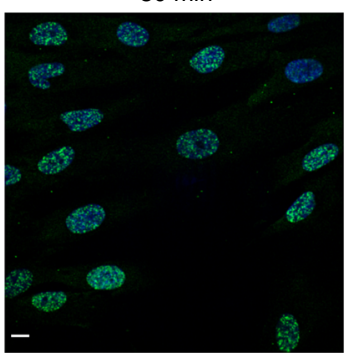

$24 \mathrm{~h}$

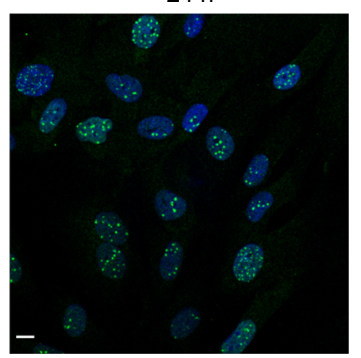

C

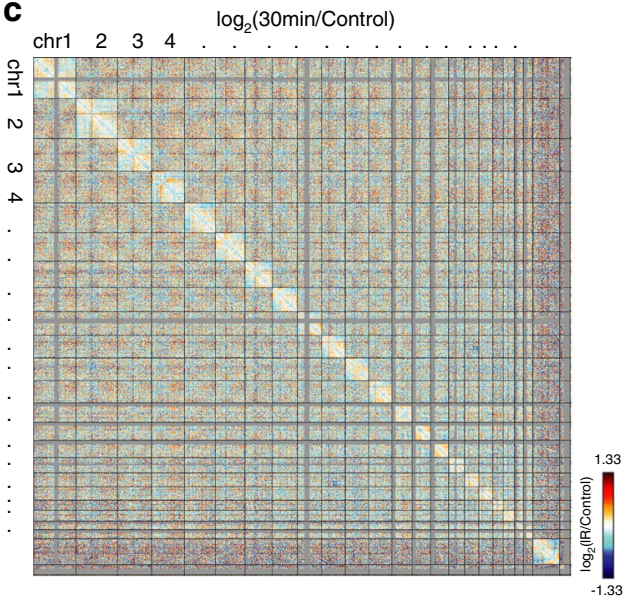

d

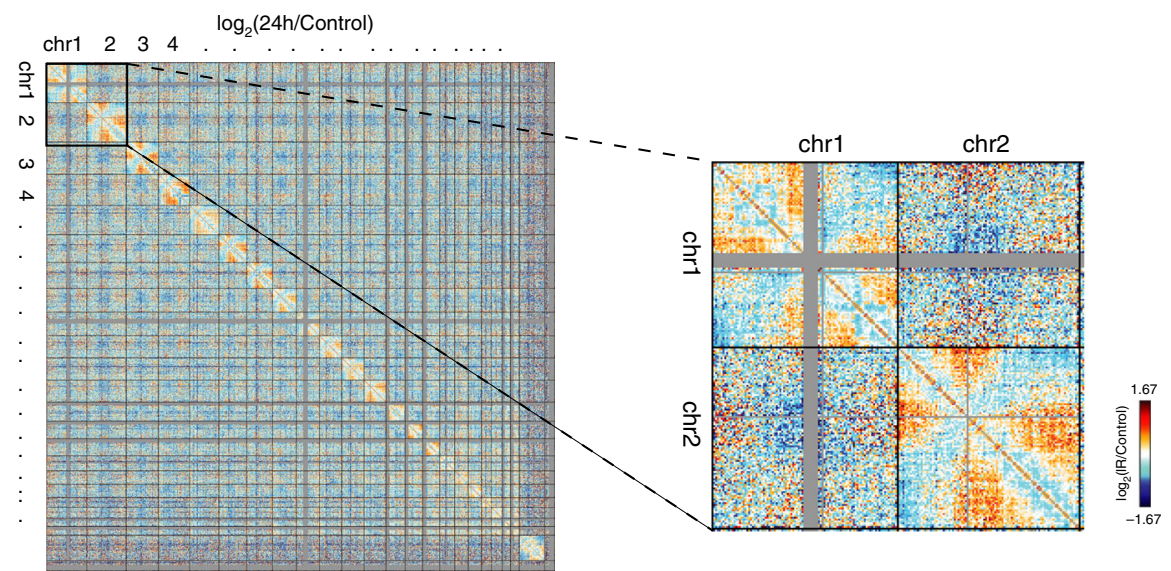

Fig. 1 Subtle changes to genome organization in IR exposed BJ-5ta skin fibroblasts. a BJ-5ta stained with $\gamma \mathrm{H} 2 \mathrm{AX}$ (green) and DAPI (blue) before (Control) or after (30 min and $24 \mathrm{~h}$ ) exposure to $5 \mathrm{~Gy}$ X-rays. Scale bar: $10 \mu \mathrm{m}$. b Genome-wide contact heatmap for BJ-5ta nonirradiated (lower left) or $30 \mathrm{~min}$ after IR (upper right) in $2.5 \mathrm{Mb}$ bins. c log2 (30 min post-IR/Control) contact heatmap in $2.5 \mathrm{Mb}$ bins. $\mathbf{d} \log 2$ (24 h post-IR/Control) contact heatmap in $2.5 \mathrm{Mb}$ bins. Inset reveals loss of interactions between chromosome arms and decrease in telomere-telomere interactions on chromosomes 1 and 2 .

information). We perform Hi-C on populations of 5 million cells, and since each cell is likely to harbor $100^{-} 200$ DSBs after 5 Gy radiation, we are measuring aggregate genome structure effects of a set of $\sim 1$ billion DSBs across the cell population, along with other forms of non-DSB damage that can be created by $\mathrm{IR}^{36,37}$. Strikingly, despite the observation of obvious DSB induction and repair events detected by $\gamma-\mathrm{H} 2 \mathrm{AX}$, only subtle changes were detected in whole-genome contact maps after $30 \mathrm{~min}$ (Fig. 1b) or $24 \mathrm{~h}$ (Supplementary Fig. 4a) after IR exposure when compared with nonirradiated control cells. Chromosome territories were maintained at $30 \mathrm{~min}$, and there is no evidence of recurrent translocations, insertions, or deletions in the cell population at 30 $\min$ or $24 \mathrm{~h}$ post-IR. However, the log ratio of contact frequency at $30 \mathrm{~min}$ post-irradiation compared to control shows a decrease in interactions between chromosome arms. This suggests a separation of chromosome arms after irradiation, which could indicate a loss of a Rabl-like configuration ${ }^{38}$. (Fig. 1c). The decrease in interactions between chromosome arms persists at 24 $h$, accompanied by an even greater loss of interactions between telomeres and some increase in interaction between telomeres and centromeres (Fig. 1d). Hi-C experiments were also carried out in BJ1 hTERT cells as an independent biological replicate. This is also an immortalized BJ fibroblast cell line made from a different clonal population than BJ-5ta. The BJ1 Hi-C results cluster well with BJ-5ta experimental data (Supplementary Fig. 5), and we observe similar changes to interaction patterns in these cells: the loss of interactions between chromosome arms (Supplementary Fig. 4c) as well as subtle changes at $30 \mathrm{~min}$ 
a

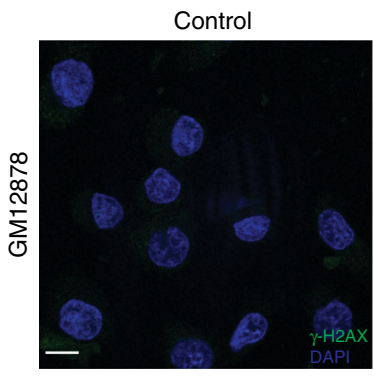

b

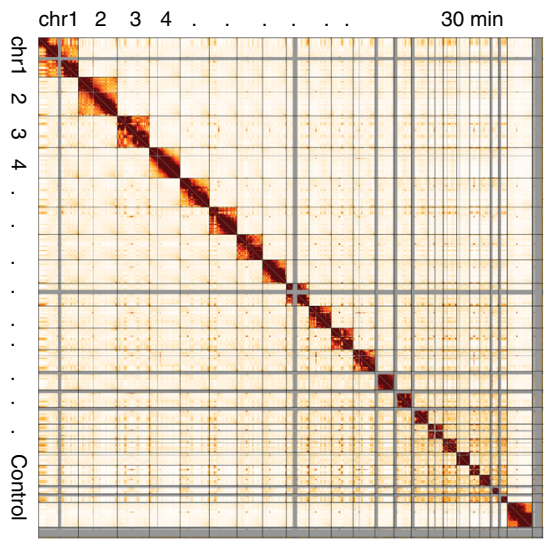

$30 \mathrm{~min}$

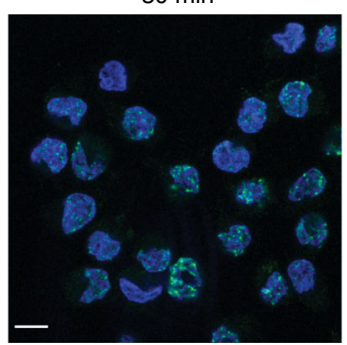

$24 \mathrm{~h}$

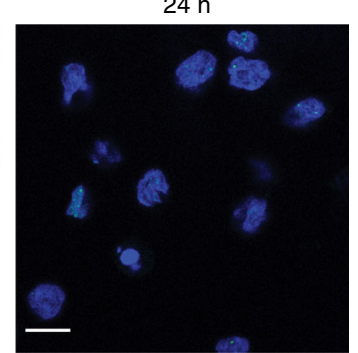

C

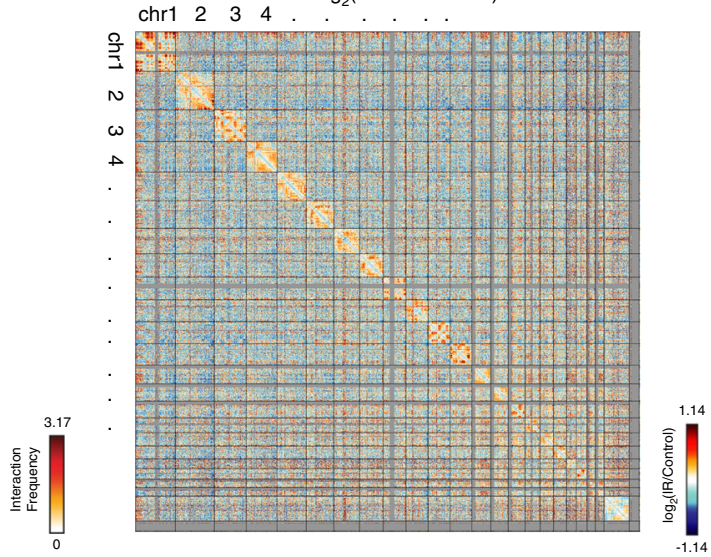

d

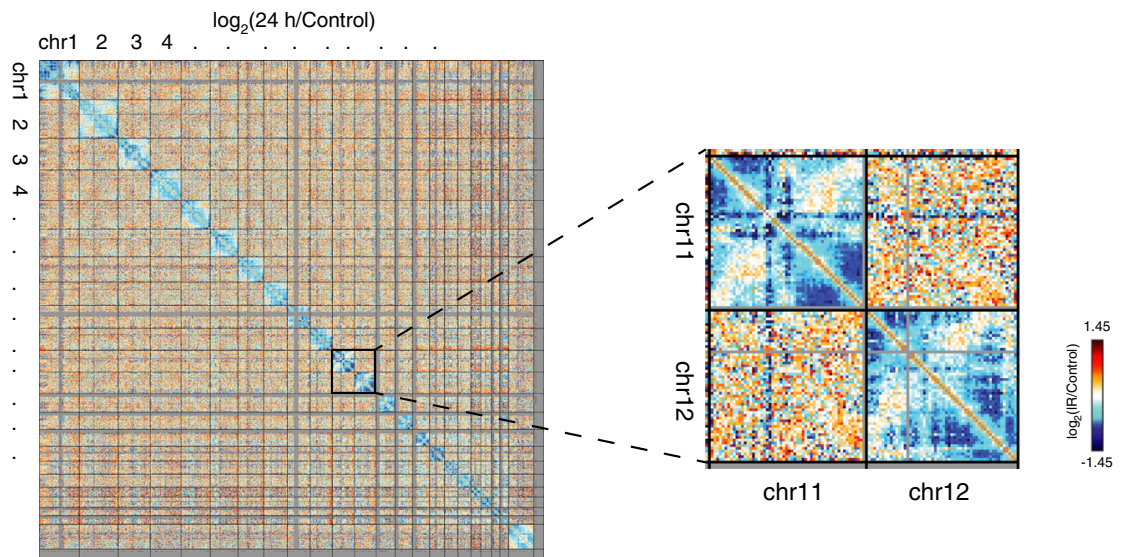

Fig. 2 GM12878 exhibits more evident changes to genome organization after IR. a GM12878 stained with $\gamma \mathrm{H} 2 \mathrm{AX}$ (green) and DAPI (blue) before or after exposure to $5 \mathrm{~Gy}$ X-rays. Scale bar: $10 \mu \mathrm{m}$. b Genome-wide contact heatmap of GM12878 in nonirradiated (left) or $30 \mathrm{~min}$ after IR (right) in $2.5 \mathrm{Mb}$ bins. $\mathbf{c} \log 2(30 \mathrm{~min}$ post-IR/Control) contact heatmap in $2.5 \mathrm{Mb}$ bins. $\mathbf{d} \log 2(24 \mathrm{~h}$ post-IR/Control) contact heatmap in $2.5 \mathrm{Mb}$ bins. Inset reveals example patterns of intrachromosomal interactions that are lost after exposure to IR.

becoming more apparent at $24 \mathrm{~h}$ post-IR (Supplementary Fig. $4 b, c)$.

Lymphoblastoid cells exhibit more evident genome architecture changes after IR. We next examined whether cell-typespecific differences exist in IR-induced organizational changes by comparing the results just discussed for human fibroblasts with results for a lymphoblastoid cell line (GM12878). As stated before, X-ray induced DSB induction and repair were monitored at $30 \mathrm{~min}$ and $24 \mathrm{~h}$ post-IR exposure by measuring $\gamma-\mathrm{H} 2 \mathrm{AX}$ using immunofluorescent and western blot techniques. As in BJ-5ta, an elevated level of $\gamma$-H2AX protein was observed in GM12878 cells at $30 \mathrm{~min}$ post-IR with a subsequent decline to baseline level at $24 \mathrm{~h}$ post-IR (Fig. 2a and Supplementary Fig. 6a, b). We then performed Hi-C on GM12878 cells exposed to 5 Gy X-ray after
$30 \mathrm{~min}$ and $24 \mathrm{~h}$. While $\mathrm{Hi}-\mathrm{C}$ replicates showed high correlation (Supplementary Fig. 5), we chose to treat replicates independently throughout subsequent analyses to provide independent corroboration of potential genome structure changes. Indeed, as discussed below, some features of contact changes were reproducible and others were not, suggesting that validating observations separately in multiple replicates is important, and that $\mathrm{Hi}-\mathrm{C}$ datasets should not always be pooled immediately after a single reproducibility statistic is calculated.

Much like the BJ-5ta cells, GM12878 did not exhibit any recurrent translocations, insertions, or deletions in genome structure (Fig. 2b and Supplementary Fig. 7a). However, GM12878 cells exhibit a more variable response at $30 \mathrm{~min}$ postirradiation. While there was an increase in the intrachromosomal interactions in both replicates, patterns of interactions observed were not consistent (Fig. 2c and Supplementary Fig. 7b). Both 
replicates exhibited a loss of intrachromosomal interactions at $24 \mathrm{~h}$ of post-exposure, and this loss of interactions was more pronounced in lymphoblastoid cells relative to fibroblasts. Also, GM12878 cells compared to fibroblasts showed more patterns of localized loss of intrachromosomal interactions at specific regions after $24 \mathrm{~h}$ of IR exposure (Fig. 2d). These patterns of lost contacts were consistent between independent replicates (Supplementary Fig. 7c).

Interestingly, many of the regions which show increased interactions at $30 \mathrm{~min}$ decreased in interactions after $24 \mathrm{~h}$. Some of the regions involved in contact loss are notably gene dense, but further work will be needed to investigate whether other properties, like combinations of histone modifications, help explain these regions of contact change after IR.

Compartment identity is unchanged despite decrease in distal interactions after IR exposure. Since we observed changes in intrachromosomal interactions in whole-genome contact maps, we next examined higher resolution contact maps of individual chromosomes. These maps showed a depletion of interactions as distance from the diagonal increases after X-ray irradiation. This loss of distal interactions was quantified by scaling plots, which showed a loss of mid-to-long range interactions after $24 \mathrm{~h}$ in both the BJ-5ta and GM12878 cell lines (Fig. 3a, b and Supplementary Figs. $7 \mathrm{~d}$ and 8 ).

Individual chromosome contact maps showed no apparent changes in the plaid contact patterns between IR and control conditions, suggesting that $\mathrm{A} / \mathrm{B}$ compartment identity is not affected by IR (Fig. 3c, d) ${ }^{31}$. The A and B compartment pattern captures the spatial segregation of euchromatin and heterochromatin within the nucleus. It has been previously shown that heterochromatin and euchromatin are differentially affected by DNA damaging irradiation and have different DNA repair responses ${ }^{12,13}$. Despite this, we observed few instances of compartment identity switches $(<\sim 1 \%)$ in both cell types and conditions as shown in both contact maps and eigenvector compartment tracks (Fig. 3c-f and Supplementary Fig. 9a. b). Even if compartment identity does not change, the strength of interactions within existing compartments can change, so we assessed changes in compartment strength after X-ray irradiation.

Saddle plots of compartment strength changes show varied and generally weak changes in compartment strength that were not consistent between replicates of the experiment (Supplementary Fig. 10). Therefore, we concluded that the strength of interactions within compartments was not a feature consistently affected by IR and DNA repair.

TAD boundary strength and CTCF loops increase after exposure to IR. The chromosome architectural proteins CTCF and cohesin are recruited to sites of DNA damage ${ }^{19}$. CTCF occupancy increases both at binding sites flanking $\gamma$-H2AX domains ${ }^{18}$ and at sites of DNA lesions ${ }^{17}$ after DNA damage is induced. In addition to their role in the DNA damage response, CTCF and cohesin are known to be important for the formation of TADs, and alterations of CTCF and cohesin can lead to an overall change in local genome topology ${ }^{39}$. As previously mentioned, TADs have been identified as important for the spatial organization of gene regulation: enhancers and promoters are more likely to communicate within the same TAD and less likely to interact across TAD boundaries ${ }^{27,40}$. The current model for TAD formation is that loop extrusion by the cohesin complex dynamically brings together genomic regions within TADs until extrusion is blocked by a boundary protein such as $\mathrm{CTCF}^{22,23}$. But, it has not been investigated whether DNA damage and repair would weaken TAD boundaries as damaged sites rearrange or randomly diffuse, strengthen TAD boundaries, or leave them unchanged.
To assess whether TADs are affected after IR, we identified TAD boundaries and calculated the boundary strength (degree of contact separation between TADs) for each boundary using both the InsulationScore ${ }^{41}$ and the Hicratio method ${ }^{42}$. Both methods determined that TAD boundary strength increases in both BJ-5ta and GM12878 cells $24 \mathrm{~h}$ after IR (Fig. 4a, b and Supplementary Fig. 11a, b). One inconsistency that was observed between the two methods was the response at $30 \mathrm{~min}$ post-IR in GM12878. In contrast to BJ-5ta, which had an increase in boundary strength in both methods after $30 \mathrm{~min}$, GM12878 shows an increase as measured by the InsulationScore method but a decrease by the Hicratio method. This may be due to a subtle difference in the way that the algorithm detects TADs, so we focus on results that were consistent between both methods. To visualize the alterations in contacts around TAD boundaries, we plotted the average interaction profile around TAD boundaries called by the InsulationScore and Hicratio method (Fig. 4c, $d$ and Supplementary Fig. 10a-d). We observe a decrease in interactions across TAD boundaries (indicating increased TAD separation) after exposure to IR in all conditions except, again, in GM12878 at $30 \mathrm{~min}$ post-IR. This divergence at $30 \mathrm{~min}$ between cell types may reflect different rates of DNA damage response and repair in different cell types, but the consistent effects on TAD strength at $24 \mathrm{~h}$ across cell types and replicates suggests that this reflects a more fundamental feature of DNA damage response. The TAD boundary strengthening is observed not only in aggregate, but is also detectable at specific loci (Fig. 4e and Supplementary Fig. 13). To test the hypothesis that TAD boundary strengthening might occur primarily in euchromatin or heterochromatin, we analyzed A or B compartment regions of the genome separately. We observe that both compartments exhibit increases in TAD boundary strength after IR (Supplementary Fig. 14).

Notably, we observe of a similar increase in TAD boundary strength $24 \mathrm{~h}$ after IR in both lung fibroblasts (MRC-5) and an independent line of foreskin fibroblasts (BJ1-hTERT) (Supplementary Fig. 11a, b). This supports the idea that the increase of TAD boundary strength is a general phenomenon, perhaps related to the dual roles of CTCF and cohesin in DNA damage response and genome architecture. The observation of this phenomenon in both fibroblasts and lymphoblasts also makes it less likely that the TAD strengthening observed is simply an effect of cell cycle state. BJ cells, as noted, are predominantly in G1/G0 phase before irradiation and become more uniform after irradiation. In contrast, GM12878 cells are still cycling at the time of irradiation and maintain similar distributions of cells in G1 and G2 at $24 \mathrm{~h}$ post-IR (Supplementary Fig. 1b). Despite this difference, TAD strengthening is observed in both cell types. The TAD strengthening observed in BJ-5ta fibroblasts can be blocked by treatment with KU-55933, an ATM kinase inhibitor (Fig. 4a, b). As explored further below, this result suggests DNA repair pathways lead to TAD boundary strengthening (Fig. 4a, b).

If TAD boundaries are reinforced after IR because of increased recruitment of CTCF after DNA damage, we would expect that this might also increase contact frequency within CTCF-mediated loops. To test this hypothesis, we used the previously annotated set of loops from high resolution GM12878 Hi- C data, specifically analyzing those with CTCF motifs at both loop anchors ${ }^{43}$. We aggregated the interaction patterns across all loop regions and found that CTCF loops indeed show an increase in average strength after exposure to IR in both fibroblasts and lymphoblasts (Fig. 4f, g).

ATM is necessary for IR-induced genome architecture changes. Changes observed in the 3D genome structure after IR exposure could be a passive side effect of DNA DSBs. However, the TAD 
a

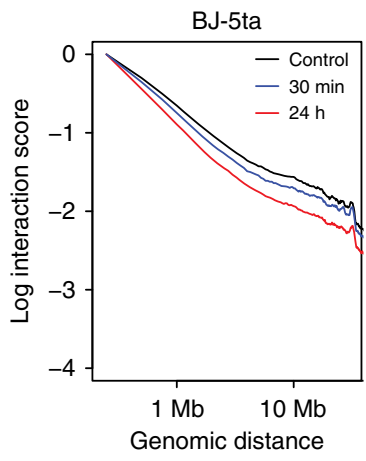

C chr4

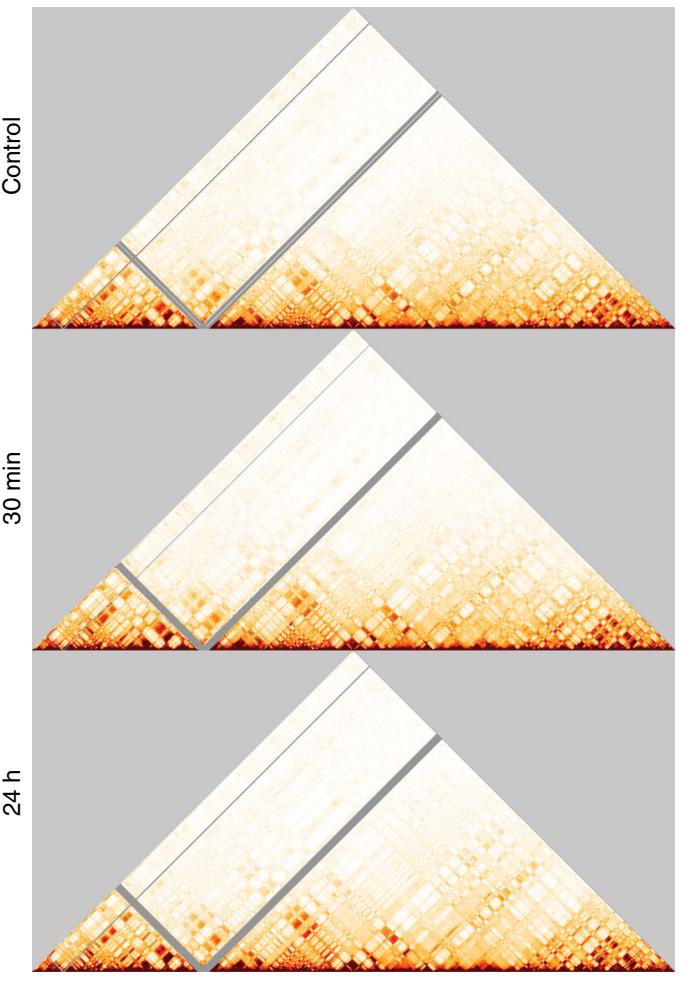

e

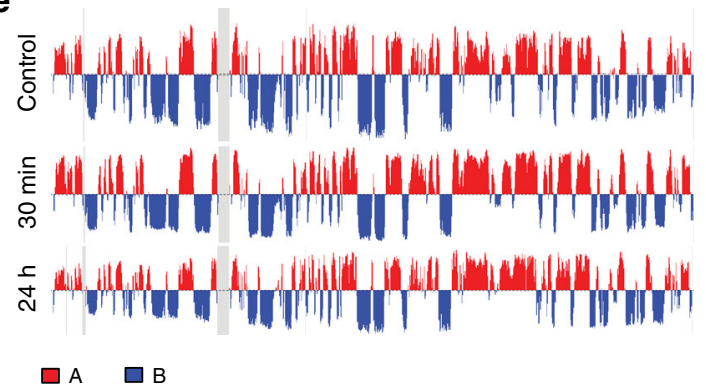

b

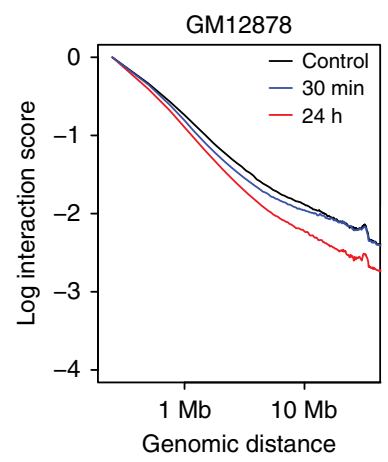

d

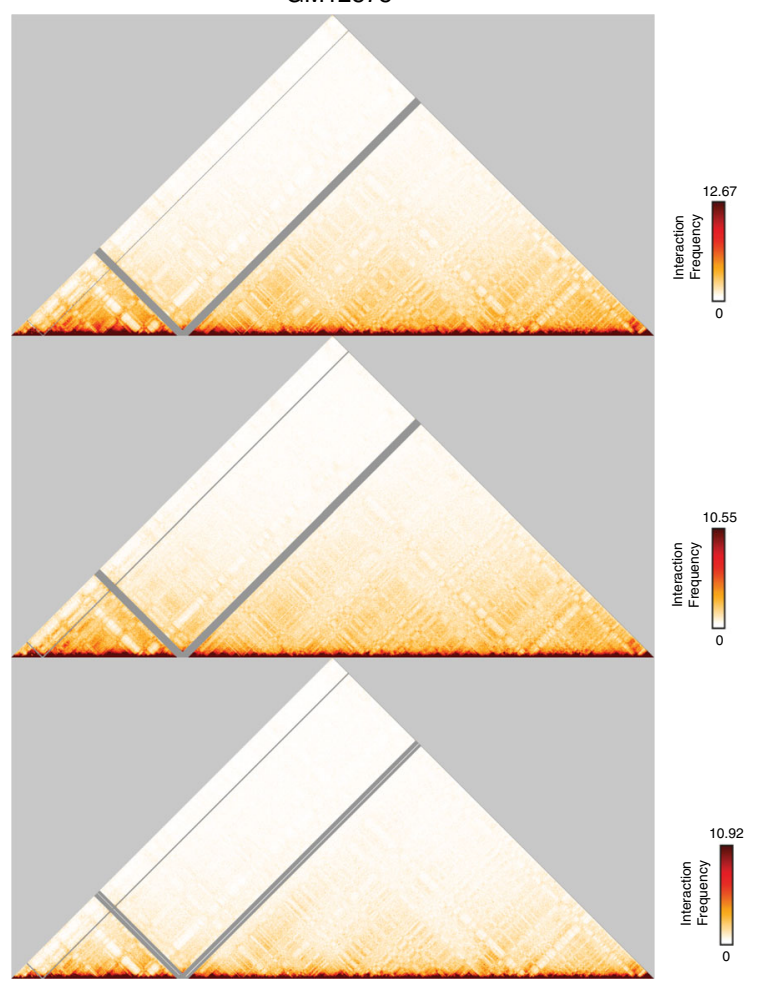

f

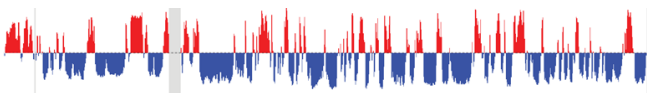

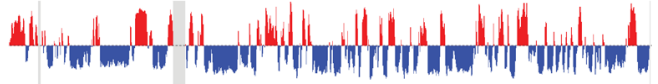

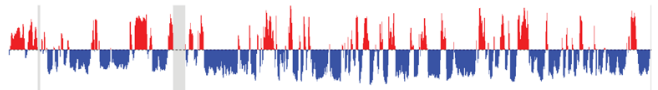

Fig. 3 Loss of distal contacts, compartment maintenance after IR. a, b Scaling plots showing average decay of contacts with distance across all chromosomes at a $250 \mathrm{~kb}$ bin size in BJ-5ta (a) or GM12878 (b) cells for control, 30 min post-IR, and $24 \mathrm{~h}$ post-IR. c, d $250 \mathrm{~kb}$ binned Hi-C interaction heatmaps for chromosome 4 in BJ-5ta (c) and GM12878 (d) cells in control (Top), 30 min post-IR (Middle), and $24 \mathrm{~h}$ post-IR (Bottom). e, f Plots of the first eigenvector from principal component analysis of chromosome 4 in BJ-5ta (e) and GM12878 (f) cells in control (Top), 30 min post-IR (Middle), and $24 \mathrm{~h}$ post-IR (Bottom).

boundary and CTCF loop strengthening that we observe are the opposite of what would be expected from a passive mixing of broken chromosomes, so we hypothesized that these changes are dependent on the cell's active response to DNA damage. Ataxia telangiectasia mutated (ATM), a PI3K-like protein kinase, plays a key role in the repair of DSBs through phosphorylation of number of downstream targets ${ }^{44-46}$. Patients with mutations in the ATM gene have an increased likelihood of several types of cancer, including breast cancer, leukemias, and lymphomas ${ }^{47,48}$. ATM phosphorylates the histone variant $\mathrm{H} 2 \mathrm{AX}$, which results in 
a

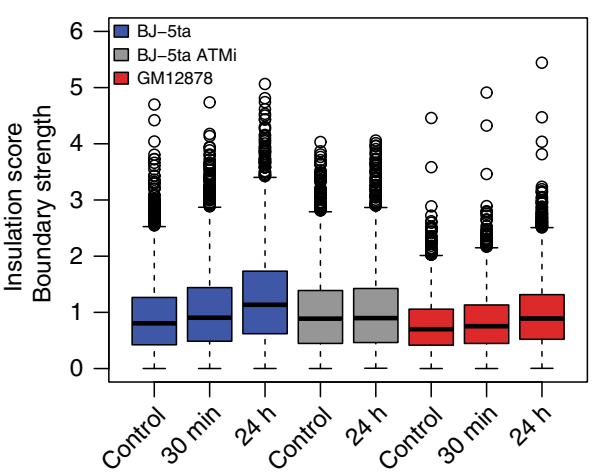

b

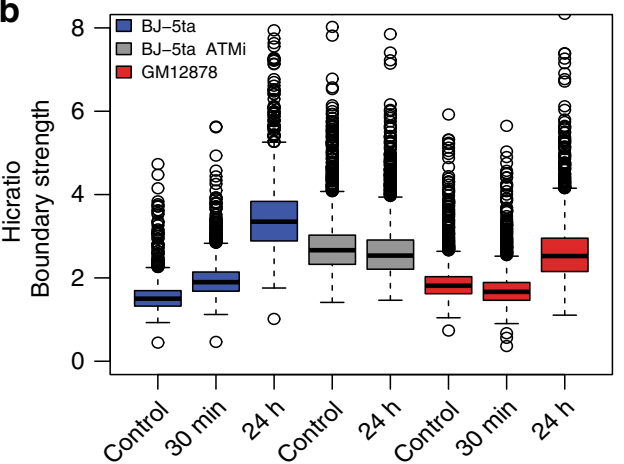

C

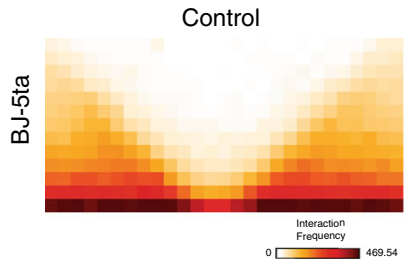

d

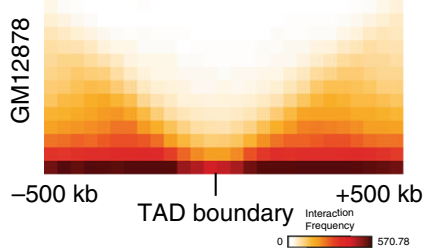

e

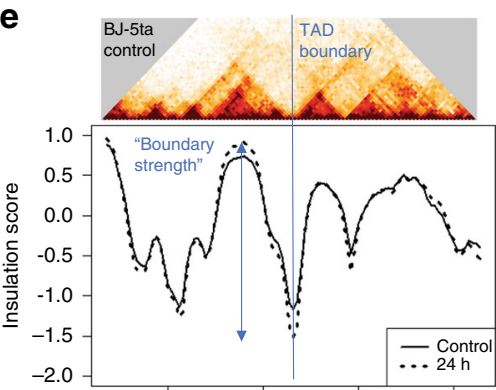

Chr2 (bp): $2.9 \mathrm{e}+073.0 \mathrm{e}+073.1 \mathrm{e}+073.2 \mathrm{e}+07$ $\log _{2}(30 \mathrm{~min} /$ Control $)$

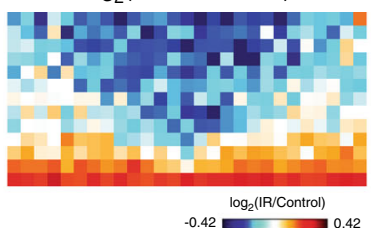

$-0.42 \log _{2}$

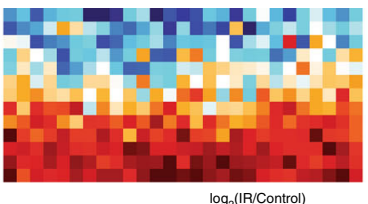

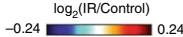

f

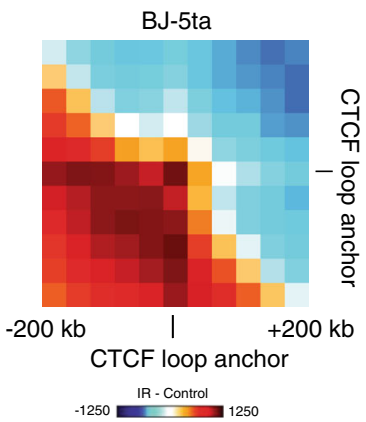

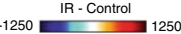

$\log _{2}(24 \mathrm{~h} /$ Control $)$
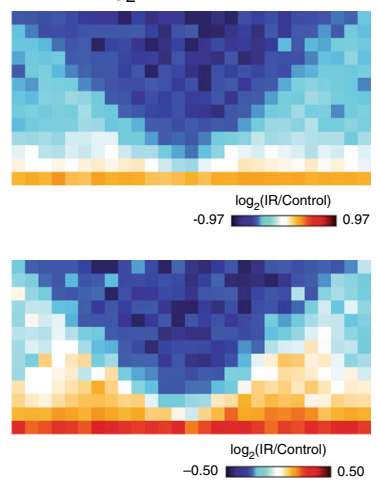

g

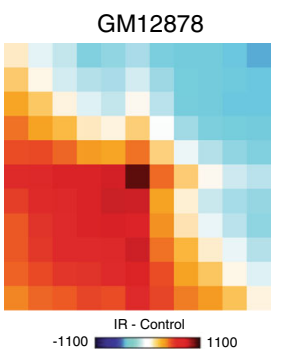

Fig. 4 TAD boundary strength and CTCF loop strength both increase after IR. a, b TAD boundary strength boxplots calculated using both the InsulationScore (a) (Number of TAD boundaries (N) BJ-5ta $=1232, \mathrm{BJ}-5 \mathrm{ta}-\mathrm{ATMi}=2010, \mathrm{GM} 12878=1091$ ) and Hicratio (Number of TAD boundaries (N) $\mathrm{BJ}-5 \mathrm{ta}=1298, \mathrm{BJ}-5 \mathrm{ta}-\mathrm{ATMi}=2868, \mathrm{GM} 12878=1878)(\mathbf{b})$ methods for both BJ-5ta (blue) and GM12878 (red) cells. Gray represents the lack of TAD strength increase at $24 \mathrm{~h}$ after IR when BJ-5ta cells were treated with ATM inhibitor. Boxes represent the upper and lower quartiles with the center line as the median. Upper whiskers extend 1.5 $\times$ IQR beyond the upper quartile, and lower whiskers extend either $1.5 \times$ IQR below the lower quartile or to the end of the dataset. A Wilcoxon signed rank test with continuity correction determined that both BJ-5ta and GM12878 control boundary strength distributions were statistically less than their $30 \mathrm{~min}$ and $24 \mathrm{~h}$ counterparts $\left(p<2.2 \times 10^{-16}\right)$. c, d Aggregate contact maps ( $40 \mathrm{~kb}$ bins) at TAD boundaries called by the InsulationScore method with strength greater than 1 in BJ-5ta (c) and GM12878 (d): Control (Left), $\log 2(30$ min/control) (Middle), and log2(24 h/ Control) (Right). e Example of strengthened TAD boundary. Top-BJ-5ta control Hi-C contacts around a TAD boundary from chr2. BottomInsulationScore profile across the region showing the sharper dip in $24 \mathrm{~h}$ (dotted) vs. control (solid) at the TAD boundary. $\mathbf{f}, \mathbf{g}$ Subtracted heatmaps for $24 \mathrm{~h}$ minus Control aggregate interactions around loop anchors with CTCF sites (3851 total loops) for BJ-5ta (f) and GM12878 (g).

the recruitment of proteins involved in DNA damage response, including genome architecture proteins CTCF and cohesin ${ }^{49}$. Interestingly, the observation that $\gamma$-H2AX foci are bound by CTCF and are approximately the size of TADs has led several groups to hypothesize that ATM-mediated repair is restricted to TADs ${ }^{29,50,51}$. To evaluate the role of ATM in the 3D genome response to X-ray irradiation, we performed similar IR experiments on ATM mutant fibroblasts immortalized with human telomerase reverse transcriptase (ATM-hTERT) as we did for previous cell types. Immunofluorescence imaging confirmed the faulty DNA repair pathway in these cells, shown by a lower mean fluorescence intensity of $\gamma$-H2AX after IR than was observed in BJ-5ta fibroblasts (Fig. 5a and Supplementary Fig. 15). The presence of $\gamma$-H2AX at all after IR in the ATM mutants suggests that an alternate mechanism, likely the ATR pathway, in part compensates for the deficiency in $\mathrm{ATM}^{52}$.

We performed Hi-C on ATM-hTERT cells before (Control) and $24 \mathrm{~h}$ post-irradiation as a comparison to the condition in 
a

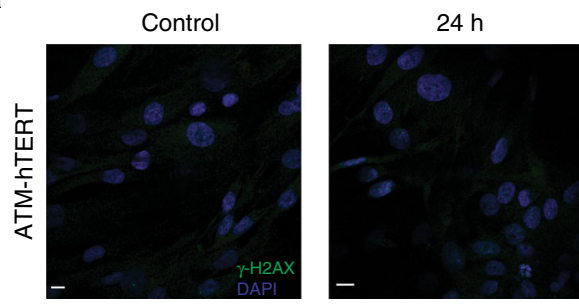

C

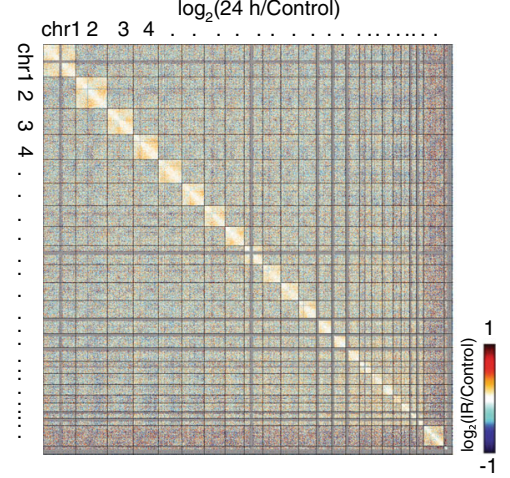

e chr4

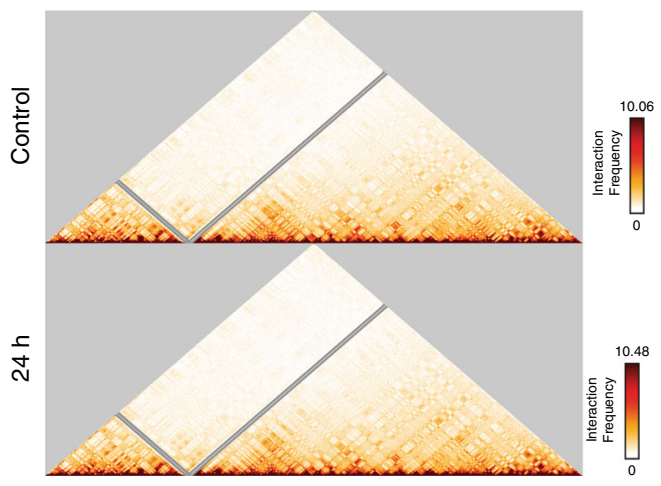

을

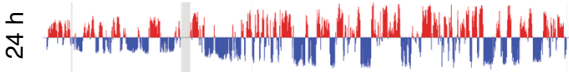

$\square \mathrm{A} \quad \square \mathrm{B}$ b
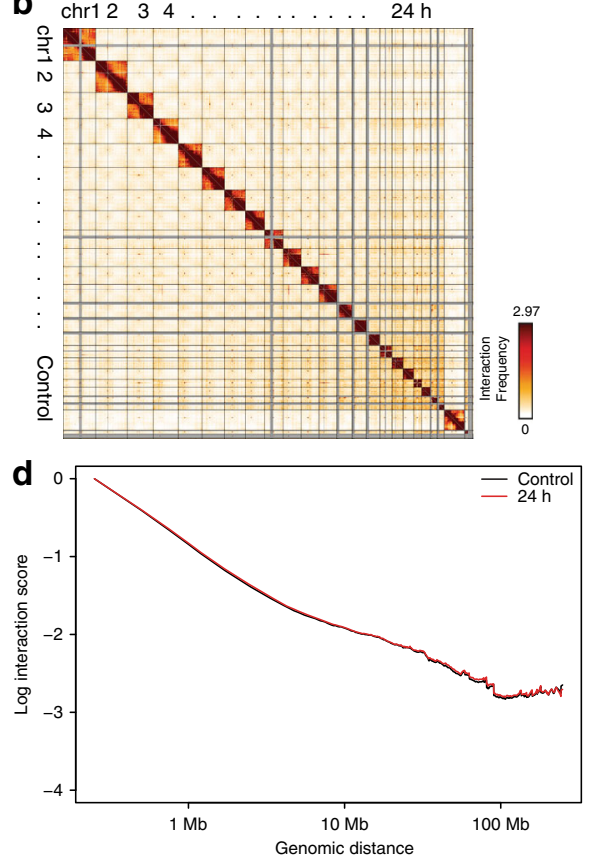

$\mathbf{f}$

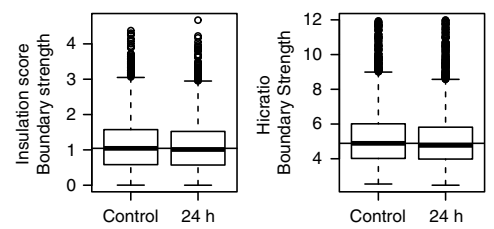

9

$\log _{2}(24 \mathrm{~h} /$ Control $)$

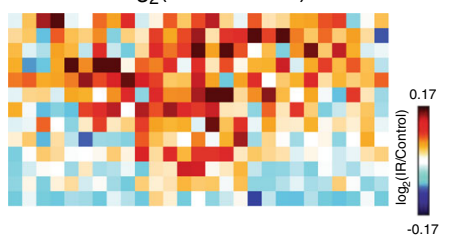

h

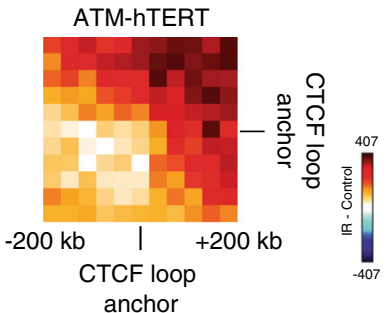

Fig. 5 ATM is necessary for 3D genome organization change after IR exposure. a ATM mutant cells stained with $\gamma \mathrm{H} 2 \mathrm{AX}$ (green) and DAPI (blue) after before (Control) or after exposure to $5 \mathrm{~Gy}$ X-rays. Scale bar: $10 \mu \mathrm{m}$. b Genome-wide contact heatmap of ATM mutant cells in nonirradiated (lower left) or $24 \mathrm{~h}$ after IR (upper right) in $2.5 \mathrm{Mb}$ bins. c log2(24 h post-IR/Control) contact heatmap in $2.5 \mathrm{Mb}$ bins. $\mathbf{d}$ Scaling plots showing average decay of contacts with distance across all chromosomes at a $250 \mathrm{~kb}$ bin size in ATM mutant cells for control and $24 \mathrm{~h}$ post-IR. e $250 \mathrm{~kb}$ bin size Hi-C interaction heatmaps for chromosome 4 in ATM mutant cells in control (Top) and $24 \mathrm{~h}$ post-IR (Bottom) show little difference in compartment as evidenced by little change to A (red) and B (blue) compartmentalization in the first principle component (below heatmaps). $\mathbf{f}$ TAD boundary strength boxplots calculated using both the InsulationScore (Number of TAD boundaries (N) ATM-hTERT =1311) and Hicratio (Number of TAD boundaries (N) ATM-hTERT = 1674) methods for ATM mutant cells. A one-tailed Wilcoxon signed rank test showed that the $24 \mathrm{~h}$ sample boundaries were not statistically increased compared to controls. Boxes represent the upper and lower quartiles with the center line as the median. Upper whiskers extend $1.5 \times I Q R$ beyond the upper quartile, and lower whiskers extend either $1.5 \times$ IQR below the lower quartile or to the end of the dataset. $\mathbf{g}$ Contact maps were aggregated at TAD boundaries called by InsulationScore with strength greater than 1 and then these maps are compared by log2( $24 \mathrm{~h}$ post-IR/Control). $\mathbf{h}$ Subtraction of $24 \mathrm{~h}$ minus Control aggregate CTCF-anchored loop contact maps in these ATM mutant cells. 
which the largest changes in genome organization occurred in BJ5ta and GM12878 cells. Like both repair-proficient cell types, no recurrent translocations were observed in ATM-hTERT cells at $24 \mathrm{~h}$ post-IR (Fig. 5b). The log ratio of Hi-C contact maps $24 \mathrm{~h}$ post-IR vs. control showed only modest changes in contacts after IR in these ATM mutant cells (Fig. 5c). We observed no change between post-IR and control scaling plots suggesting that the reduction of distal interactions in BJ-5ta and GM12878 cells is dependent on DNA damage response and repair. As in BJ-5ta cells, A/B compartment identities in ATM mutant cells are maintained $24 \mathrm{~h}$ after exposure to IR (Fig. 5e). To evaluate TAD strength changes in ATM mutant cells after X-ray, we again used both the InsulationScore and Hicratio methods. In contrast to the increased TAD boundary strength observed in all DNA repair proficient cells after X-ray, we observe a slight decrease in TAD boundary strength in ATM mutant cells after IR (Fig. 5f). We confirmed that there was an increase in interactions on average across TAD boundaries after X-ray in the ATM mutant cells by plotting the average interaction profile around TAD boundaries (Fig. 5g). Additionally, in contrast with BJ-5ta and GM12878, aggregate interaction patterns showed no increase of CTCF loops in ATM mutant cells (Fig. 5h). These results suggest that ATM is necessary for the increased segregation of TADs after IR, and that in the absence of ATM, genomic regions have a higher chance of interacting across TAD boundaries. To ensure that these results are attributable to ATM and not an artifact of TERT immortalization or specific to one cell line, we repeated the same set of experiments using primary human fibroblasts derived from an AT patient (GM02052). We confirmed that in the absence of functional ATM, insulation between TADs remained the same or decreased (Supplementary Fig. 11a, b).

As a final confirmation of the importance of ATM to the 3D genome structure response to IR, we compared the effects of IR in a syngeneic system, treating BJ-5ta cells with $10 \mu \mathrm{M}$ KU-55933 (an ATM inhibitor) for $1 \mathrm{~h}$ and then irradiating with $5 \mathrm{~Gy}$ X-rays. This treatment abrogates the $\gamma$-H2AX signal after IR, as expected (Supplementary Figs. 16a, 17). We find that all the major effects of IR on untreated BJ-5ta cells are lost after ATM inhibition in this same cell line: there is no loss of Rabl-like chromosome arm contacts, no decrease in distal chromosome contacts, and no increase in TAD boundary or CTCF loop strength.

3D genome response to IR is delayed in ATM mutants and persists in wild-type fibroblasts. The persistence of changes observed in the TAD strength at $24 \mathrm{~h}$ after IR exposure in DNA repair proficient cells is striking, given that most DNA damage is repaired/misrepaired by this time point. Over an even longer time course after IR, cells that have failed to repair properly will often die and be eliminated from the population, selecting for cells that can continue to proliferate after repair. To determine the longterm effects of IR and the subsequent repair of DSBs on the $3 \mathrm{D}$ genome, we irradiated BJ-5ta and ATM-hTERT fibroblasts with $5 \mathrm{~Gy} \mathrm{X}$-rays and then split the cells into sub- confluent densities after $24 \mathrm{~h}$. Cells were allowed to recover and proliferate until confluent ( 4-5 days). Hi-C experiments on the recovery condition from both cell lines showed that BJ-5ta fibroblasts maintained the loss of interactions at telomeric regions in large chromosomes observed at $30 \mathrm{~min}$ and $24 \mathrm{~h}$ post-IR but showed a decrease in interactions at the centromeric regions among most chromosomes. Interactions that changed $24 \mathrm{~h}$ post-irradiation are even more apparent in recovery (Fig. 6a). In ATM mutant cells, genome-wide contacts remain largely unaffected after 5 days recovery, as at $24 \mathrm{~h}$ after IR. The notable exception was that one replicate showed the emergence of two new translocations after 5 days of recovery: between chromosomes 17 and 21 and 19 and
21 (Fig. 6b). This is consistent with the repair-deficient nature of ATM mutant cells. As at $24 \mathrm{~h}$ post-IR, mid-to-long range interactions remained decreased in BJ-5ta recovery as compared to the control (Fig. 6c). In addition, ATM mutants also had a decrease in mid-to-long range interactions after recovery (Fig. 6c), which differs from the lack of change observed after $24 \mathrm{~h}$ in these mutant cells (Fig. 5d). A/B compartment identity is maintained in both BJ-5ta and ATM mutant cells after recovery, which is consistent with data obtained from $30 \mathrm{~min}$ and $24 \mathrm{~h}$ IR responses (Fig. 6d).

TAD boundary strength measured by both InsulationScore and Hicratio methods remains higher than control 5 days after IR in BJ-5ta, suggesting that TADs maintain increased segregation long term after IR (Fig. 6e). Consistent with this result, the average TAD profile comparison showed a decrease in interactions across BJ-5ta TAD boundaries in 5-day recovery vs. control (Fig. 6f). Interestingly, ATM mutant cells also show some evidence of increased segregation of TADs after 5 days of recovery. The effect is not as strong or consistent, however, and varies by the quantification approach used (Fig. 6e).

This again suggests that IR effects on TAD boundaries in ATM mutant cells are not the same as in repair- proficient cells but indicates that there could be a longer-term convergence, in which the ATM mutant cells that survive radiation long-term eventually show increased boundary strength. (Fig. 6f and Supplementary Fig. 8d).

\section{Discussion}

The 3D architecture of the genome influences various DNA metabolic activities, including DNA repair, to ensure the maintenance of genomic stability. Although IR-induced gene mutations and chromosomal alterations have been well studied in various cell and animal model systems, IR-induced structural changes at the level of higher order chromatin organization have been given less attention.

In this study, we have identified notable and lasting effects of irradiation on genome organization within the cell population. Overall, the changes in 3D genome contacts that we observe after irradiation provide a new platform from which to consider the interplay between local chromatin structure, genome architecture proteins, TAD strength, and DNA repair after irradiation. Changes that are evident in population-averaged $\mathrm{Hi}-\mathrm{C}$ data must have been occurring consistently, or at least in a substantial minority of cells, across the cell population. Our analysis methods also required that changes occur consistently across completely independent biological replicates. On the one hand, this is a potential weakness in the sense that neither we see the changes that are only occurring in certain individual cells, nor can we link a given change to a certain specific location of DNA damage. Even if we mapped genome-wide sites of DNA damage across the population of cells, we would not expect there to be specific hotspots of damage that we could correlate with our 3D genome effects $^{53}$. But, this requirement for signals to rise above the inherent variability in each cell means that the changes we observe report consistent features of the genome structure's response to irradiation. Such features are likely to reflect biologically controlled and significant responses. It is important to note that we cannot distinguish whether these consistent changes are the aggregate of many local responses or widespread long-distance propagating responses to single breaks. However, by comparing our data to future work on the $3 \mathrm{D}$ genome response to individual breaks, we will be better able to distinguish these possibilities ${ }^{29}$. Our results, combined with other published data, do indicate that we are observing $3 \mathrm{D}$ genome responses to DNA damage specifically rather than a generic cellular stress 
a

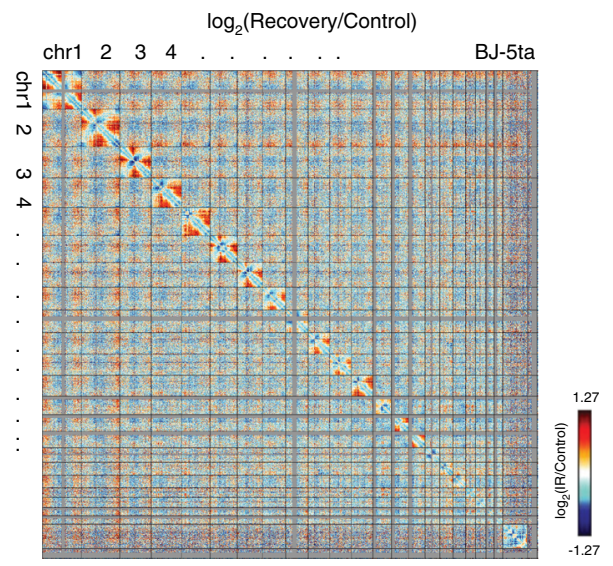

C
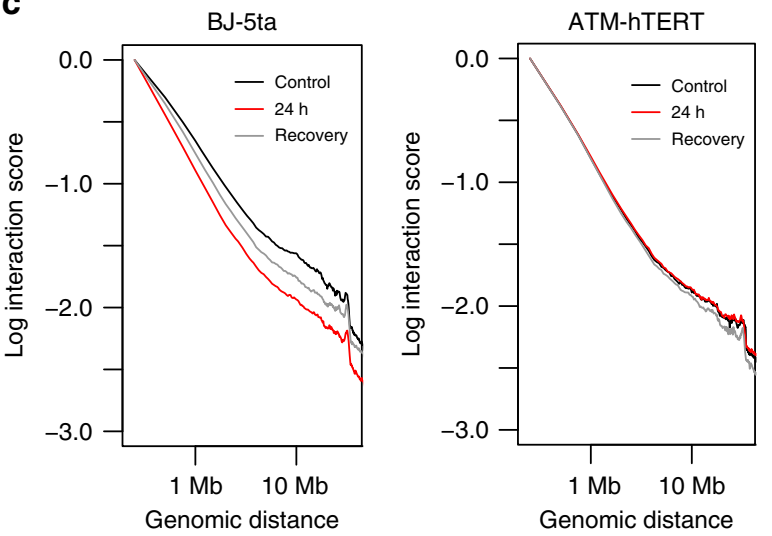

e
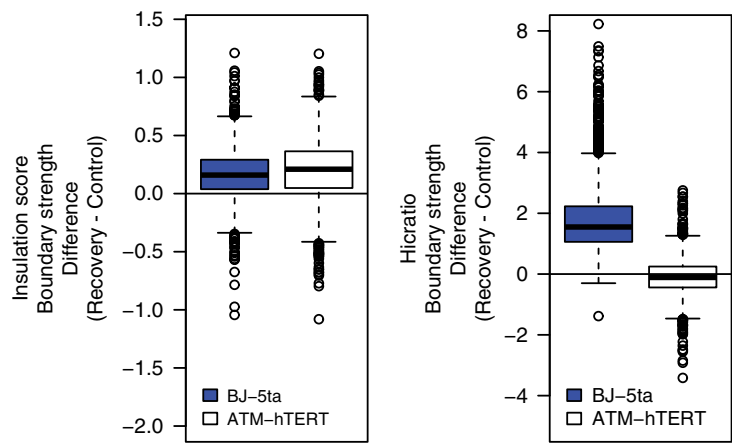

b
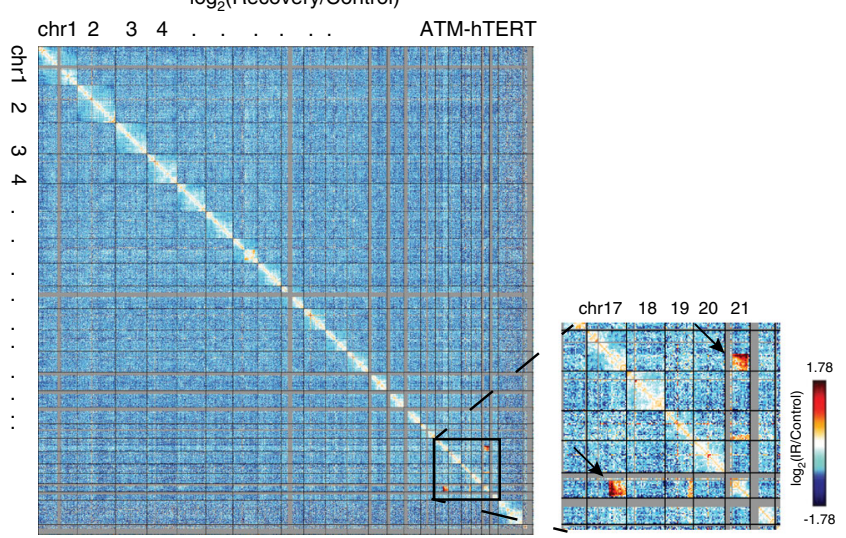

d chr4

BJ-5ta

ATM-hTERT
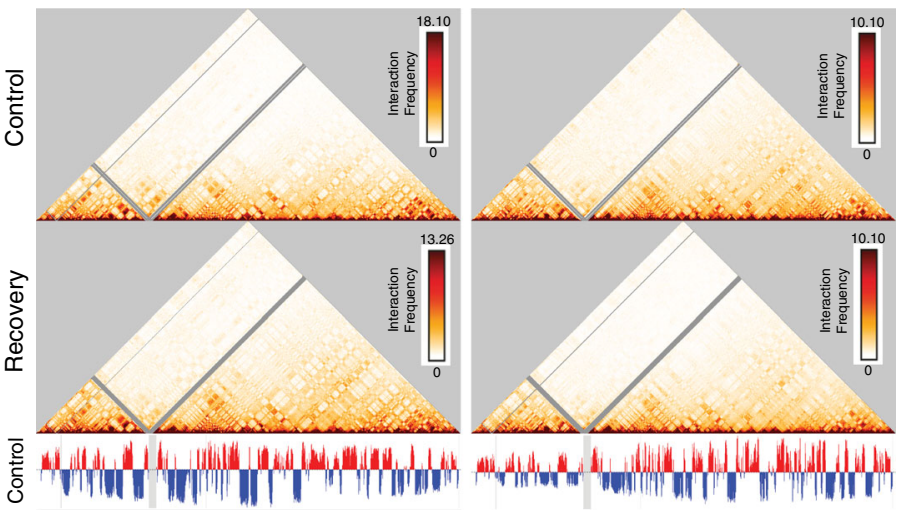

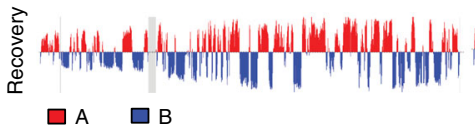

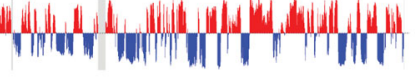

f

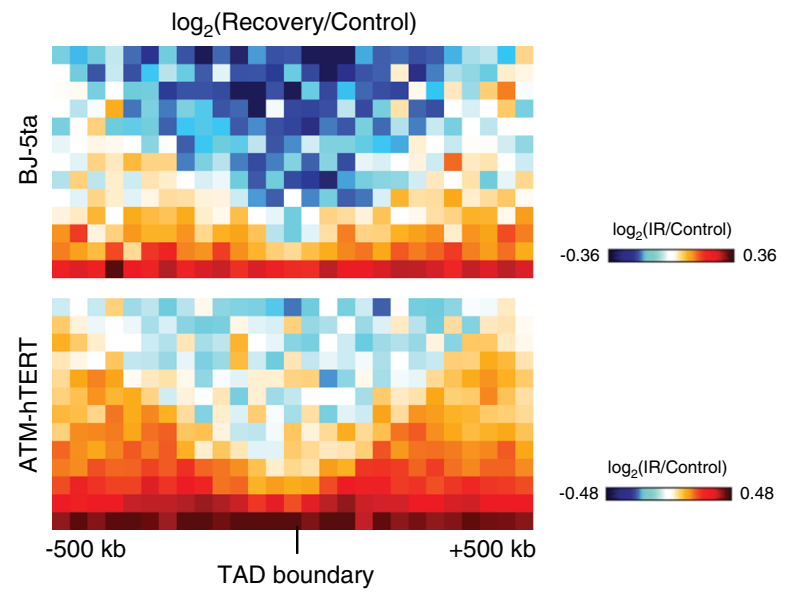

Why are certain cell types, genetic profiles, or disease states more radiosensitive than others ${ }^{57}$ ? Under highly similar culture and IR exposure conditions, we see that fibroblasts show an overall loss of chromosome arm contacts after IR while lymphoblasts show loss of interactions between specific compartment-scale genomic regions. We hypothesize that variations in initial chromosome structure and local chromatin state are a good candidate to explain some of the variations in the response to IR of different cells under different conditions. Since notable variations in initial chromosome structure occur across different cell types, in disease, and in response to different external conditions ${ }^{54,55,58}$, our results 
Fig. 6 Similarities and differences in 3D genome organization changes after $\mathbf{5}$ days recovery. a $\log 2$ (Recovery/Control) contact heatmap for BJ-5ta binned at $2.5 \mathrm{Mb}$. Inset reveals the loss of interactions between chromosome arms and decrease in telomere-telomere interactions on chromosomes 1 and 2 persists. $\mathbf{b} \log 2$ (Recovery/Control) contact heatmap in ATM mutant cells binned at $2.5 \mathrm{Mb}$. Inset indicates translocations observed between chromosomes 17 and 19 and 19 and 21 at 5 days post-IR that were not in the control ATM mutant cells. c Scaling plots showing average decay of contacts with distance across all chromosomes at a $250 \mathrm{~kb}$ bin size in BJ-5ta (left) and ATM mutant (right) cells before X-ray IR and after 5 days recovery. $\mathbf{d} 250 \mathrm{~kb}$ bin size $\mathrm{Hi}-\mathrm{C}$ interaction heatmaps for chromosome 4 in BJ-5ta control (top left), BJ-5ta recovery (bottom left) ATM mutant control (top right) and ATM recovery (bottom right). PC1 compartment identity values (below heatmaps) remain relatively unchanged 5 days post-IR. e The change in strength for each TAD boundary shared between each cell type's control and IR recovery condition was calculated by InsulationScore method (left) (Number of TAD boundaries (N) BJ-5ta $=1232$, ATM-hTERT $=1311$ ) or HiCRatio (right) (Number of TAD boundaries (N) BJ-5ta $=1298$, ATM-hTERT $=1674$ ) and the distribution of changes are represented in boxplots for BJ-5ta (blue) and ATM mutant cells (white). Boxes represent the upper and lower quartiles with the center line as the median. Upper whiskers extend $1.5 \times$ IQR beyond the upper quartile, and lower whiskers extend either $1.5 \times I Q R$ below the lower quartile or to the end of the dataset. A Wilcoxon signed rank test with continuity correction determined that both BJ-5ta and ATM mutant control distributions were significantly lower than the recovery samples $\left(p<2.2 \times 10^{-16}\right)$ for the InsulationScore approach. $\mathbf{f} \log 2$ (Recovery/Control) heatmap of InsulationScore called TAD boundaries with strength greater than 1 averaged across the genome in BJ-5ta (top) and ATM mutant cells (bottom).

support future work investigating the contribution of initial chromatin state to the changes we observe.

Most strikingly, this study revealed that an increase of TAD boundary strength after exposure to IR is consistent across cell types and that this phenomenon is dependent on functional ATM activity. Considering this observation in the light of previous research suggests that this TAD strengthening after IR may arise from CTCF and cohesin's dual roles in genome architecture and DNA damage response. Cohesin has long been known to be important in the DNA damage response and is a target of ATM $^{20,59}$. ATM signaling also increases CTCF recruitment to chromatin, CTCF sites are vulnerable to DNA breaks, and CTCF depletion increases genome instability ${ }^{17,53}$. Meanwhile, increasing evidence supports the idea that cohesin and CTCF participate in the formation of TADs by loop extrusion ${ }^{2,23,24,39}$. Thus, we propose that more cohesin complexes recruited to sites of DNA damage bring regions within TADs together more frequently as they extrude loops. As they do so, they would make H2AX phosphorylation within TADs more likely, potentially explaining the apparent coincidence of $\gamma \mathrm{H} 2 \mathrm{AX}$ foci and TADs ${ }^{14,50}$. The extruding complexes would then be blocked by increased CTCF recruitment, further decreasing interactions between TADs and leading to the boundary strength increases we see across cell types.

Why would increased TAD strength matter biologically to the cells exposed to radiation? Such increased genome structure segregation in response to DNA damage could be an important part of the cell's ability to maintain genome integrity and prevent deleterious translocations. It is known that translocations, which occur across TAD boundaries, can result in the misregulation of genes and be oncogenic, and it is known that translocation frequency is influenced by pre-existing genome contact frequency ${ }^{7,8}$. Thus, decreasing contacts across TAD boundaries in response to DNA damage would be one way to decrease the frequency of translocation events that disrupt TAD boundaries. We observe long-term persistence of TAD strengthening after IR (even at 5 days after IR, when cells have been allowed to proliferate). Notably, the cells that survive to divide are those that have not been eliminated by mitotic catastrophe due to deleterious translocations. If TAD strengthening is protective, we would predict that exposure to and recovery from one round of IR would make the cell population more resistant to deleterious effects of a second round of IR. While it is difficult to quantitate what degree of boundary strength change would have a meaningful biological impact, previous work has suggested a role for similar changes in boundary elements in biological processes such as gene dosage compensation $^{41}$.

Future work will be needed to clarify the role of chromatin state, cell cycle state, and genome architecture proteins in the responses to IR that we observe. Perturbations of chromatin state before IR exposure would reveal the impact of different initial structures on downstream effects of radiation. Similarly, measuring the effect of irradiation on cells with altered levels of CTCF or cohesin will determine whether these factors indeed affect the spread of $\gamma$-H2AX. The consistent responses of GM12878, which have subpopulations in G1, G2, and S phase, and BJ cells, which are primarily in G1, suggests that effects we observe are not entirely dependent on cell cycle state. But, future work repeating these experiments on cells synchronized in different phases of the cell cycle, where different repair pathways are favored, would better clarify the role of different repair pathways in the effects we observe. Our data supports a model in which ATM is required for the reinforcement of 3D genome organization after exposure to IR. But, the possibility remains that not only ATM, but also the subsequent signaling cascade involved in DNA repair downstream of ATM may be necessary to elicit the genome structure responses we observe in healthy cells. To understand more clearly how DNA damage pathways influence the 3D genome architecture, the role of proteins such as DNA ligases and ATR must be interrogated more thoroughly $60-65$

Additionally, future work is needed to identify the biological importance of TAD strengthening after IR. It is possible that the TAD structure effects we observe are indeed a byproduct of repair processes, but not an important or controlled component of protecting the genome from aberrations. In the future, artificially altering TAD strength, by altering concentrations of architecture proteins, would allow a more direct perturbation of the effect of TAD strength on cellular radiosensitivity and downstream effects of radiation.

\section{Methods}

Cell culture. Fibroblast cell lines include: BJ-5ta (ATCC, CRL-4001), BJ1-hTERT (kind gift from Vilhelm A. Bohr, Laboratory of Molecular Genetics, Gerontology Research Center, National Institute on Aging, National Institutes of Health, Baltimore, MD), MRC-5 (kind gift from Tim Sparer, University of Tennessee, Knoxville, Knoxville, TN), AG04405 (ATM-hTERT) (kind gift from Peter Lansdorp, University of British

Columbia, Vancouver, Canada), and GM02052 (Coriell). The lymphoblastoid cell line GM12878 (Coriell) was also used. Information about cell lines and media formulations is summarized in Supplementary Table 2. All growth media and fetal bovine serum was purchased from Corning, except for GM12878 growth media from Gibco. All fibroblas lines were passaged at a density of $80 \%$. For irradiation experiments, fibroblast lines were grown to confluency prior to X-ray exposure. For the GM12878 suspension cell line, cells were passaged at a density around 500,000 cells per $1 \mathrm{~mL}$ of medium. For irradiation experiments, GM12878 cells were grown to a density of 1 million cells per 1 $\mathrm{mL}$ of medium prior to X-ray exposure.

X-ray irradiation. Cells were irradiated with $5 \mathrm{~Gy} \mathrm{X}$-rays using a RadSource RS-2000 Biological Irradiator. X-rays were emitted with a concentration of $2 \mathrm{~Gy}$ $\min ^{-1}$ with cells being exposed for $2 \mathrm{~min}$ and 30 seconds $(160.9 \mathrm{kV} ; 25.1 \mathrm{~mA})$. After irradiation, cells were returned to an incubation chamber and kept at $37^{\circ} \mathrm{C}$ 
with $5 \% \mathrm{CO}_{2}$. Cells were collected at $0 \mathrm{~min}, 30 \mathrm{~min}$, or $24 \mathrm{~h}$ post-irradiation and fixed for the respective downstream application.

Immunofluorescence staining and image analysis. Fibroblast cells were seeded on poly-D-lysine (PDL) coated $35 \mathrm{~mm}$ coverslip dishes prior to X-ray exposure. For GM12878 cells, coverslips were sterilized and coated in $0.1 \mathrm{mg} \mathrm{mL}^{-1} \mathrm{PDL}$. GM12878 cells were X-rayed while in suspension and centrifuged onto coverslips at $800 \times g$ for $10 \mathrm{~min}$ immediately before fixation. At the appropriate timepoints, all cells were fixed in $4 \%$ formaldehyde for $20 \mathrm{~min}$ at room temperature, washed in PBS for 5 min three times, and then concurrently permeabilized and blocked in blocking buffer ( $10 \%$ goat serum, $0.5 \%$ triton in $1 \times \mathrm{PBS})$ for $1 \mathrm{~h}$ at room temperature. The primary antibody to $\gamma-\mathrm{H} 2 \mathrm{AX}$ (Abcam- ab26350, 1:500) was diluted in a 1:1 mixture of blocking buffer and PBS, and incubated overnight at $4{ }^{\circ} \mathrm{C}$. Samples were then washed three times in PBS for $5 \mathrm{~min}$ and incubated in secondary antibody (Alexa Fluor 488 goat anti-mouse, Invitrogen; R37120) for $30 \mathrm{~min}$ at room temperature, in the dark. Samples were washed as previously described and exposed to $0.1 \mathrm{mg} \mathrm{mL}^{-1}$ DAPI for one minute, followed by washing. All PBS was removed by aspiration and samples were briefly air dried. Coverslips were mounted on slides with Slow fade Diamond Antifade Mountant (Molecular Probes, S36963).

Images were acquired using a Leica SP8 confocal microscope equipped with a $\times 63$ oil immersion objective (Leica) using the same settings. Image quantification was carried out by measuring the mean fluorescence intensity of $\gamma-\mathrm{H} 2 \mathrm{AX}$ inside the nucleus divided by area of the nucleus in ImageJ. $\gamma-\mathrm{H} 2 \mathrm{AX}$ foci were counted by normalizing fluorescence to remove background and using the analyze particle function to select foci. An ordinary one-way ANOVA test was used to determine statistical significance for all image quantification.

Flow cytometry and cell cycle analysis. Cells were collected before or after irradiation and fixed with $70 \%$ ethanol for $30 \mathrm{~min}$ at $4{ }^{\circ} \mathrm{C}$. Cells were then centrifuged for $10 \mathrm{~min}$ at $800 \times g$ for $10 \mathrm{~min}$ and resuspended with Guava ${ }^{\circledR}$ Cell Cycle Reagent (Luminex, 4500-0220). 10,000 events were collected for each condition on an Attune Nxt Acoustic Focusing Cytometer using software version 2.1. Cell cycle analysis was done using Flowing Software 2.5.1 (Terho, P. Cell Imaging Core, Centre for Biotechnology, University of Turku, Finland).

Hi-C. To prepare the Hi-C libraries, at the appropriate timepoints, $\mathrm{Hi}-\mathrm{C}$ experiments were performed according to the protocol detailed in Golloshi et al. ${ }^{66}$. Briefly, 5-20 million cells were fixed with $1 \%$ formaldehyde, suspended in cell lysis buffer for permeabilization, and homogenized by douncing. Crosslinked chromatin was digested overnight with HindIII. Sticky ends were filled in with biotin-dCTP, and the blunt ends of interacting fragments were ligated together. DNA was purified by two phenol-chloroform extractions and ethanol precipitation. Biotin$\mathrm{dCTP}$ at unligated ends was removed, and the DNA was sheared to a target size of 200-400 bp by a Covaris sonicator (Covaris, M220). DNA between 100 and $400 \mathrm{bp}$ was selected for using AMPure XP beads (Beckman Coulter).

Biotinylated DNA was pulled down using streptavidin coated magnetic beads and prepared for multiplex sequencing on an Illumina platform using the NEBNext Ultra II kit (NEB, E7645S). All end preparation, adaptor ligation, and PCR amplification steps were carried out on bead bound DNA libraries.

Sequencing was carried out at the Oklahoma Medical Research Foundation Clinical Genomics Facility on an Illumina HiSeq 3000 platform with 75 bp paired end reads or a NovaSeq 6000 with 50 bp paired end reads. Sequencing reads were mapped to the reference human genome hg19, filtered, and iteratively corrected using the pipeline detailed in Imakaev et al. ${ }^{67}$. and available on github (dekkerlabcMapping).

All processed Hi-C contact maps were scaled to a sum of 1 million to allow comparisons between conditions for subsequent analyses.

ATMi Treatment. BJ-5ta fibroblasts were grown to confluency and supplemented with $10 \mu \mathrm{M}$ KU-55933 (Selleck Chemicals, S1092) for $1 \mathrm{~h}$ before irradiation. For microscopy experiments, cells were fixed before and $30 \mathrm{~min}$ after $5 \mathrm{~Gy}$ X-Rays exposure consistent with previous microscopy experiments. Cells were collected for $\mathrm{Hi}$-C before exposure to $5 \mathrm{~Gy} \mathrm{X}$-rays and $24 \mathrm{~h}$ after exposure with the previously stated $\mathrm{Hi}-\mathrm{C}$ protocol.

Hi-C reproducibility assessment. A spearman correlation-based metric was used to assess reproducibility across all $\mathrm{Hi}-\mathrm{C}$ datasets.

After taking the log of iteratively corrected interaction counts, binned at $2.5 \mathrm{Mb}$ across the entire genome, correlation maps were calculated for each individual matrix. Each entry in such a $\mathrm{Hi}-\mathrm{C}$ data correlation matrix represents the correlation between the full row and column of data at that position in the matrix.

This is like the calculation typically performed to emphasize interaction patterns over the average distance decay for compartment analysis. Then, each genome-wide correlation matrix was flattened into a vector (concatenating all columns into one single column), and all vectorized $\mathrm{Hi}-\mathrm{C}$ matrices were compared with pairwise Spearman correlation to obtain the final reproducibility metric between each pair of datasets.
The results from this approach match well with the published GenomeDISCO approach $^{68}$.

Interaction vs. distance scaling plots. Using $250 \mathrm{~kb}$ binned iteratively corrected and scaled contact matrices, we extracted contact frequencies between bins at each genomic distance, excluding the diagonal bin (zero distance). We then used a loess fit to find a smooth curve describing interaction decay vs. distance. Loess curves for each chromosome were averaged together to generate a single scaling curve representing the whole genome. The interaction frequencies were then normalized to set the maximum value (loess fit value for the minimum distance) for each dataset to 1 and then plotted on a log scale.

Compartment analysis. Principal component analysis was performed using the matrix 2compartment perl script in the cworld- dekker pipeline available on github on $250 \mathrm{~kb}$ binned matrices. The first eigenvector, which typically represents the compartment profile, was used to determine A/B compartments.

TAD boundary and insulation analysis. Topologically associating domain (TAD) boundaries were called on $40 \mathrm{~kb}$ binned matrices using two methods: the InsulationScore method, as detailed in Crane et al..$^{36}$, available as matrix2insulation in the cworld-dekker github package, and the Hicratio method, as described in Gong et $\mathrm{al}^{37}$. For the InsulationScore, a $500 \mathrm{~kb}$ insulation square size was used, and for the Hicratio method, we used $\mathrm{d}=400 \mathrm{~kb}$

Insulation contact maps in main figures were generated by averaging all called TAD boundaries with an insulation score greater than one as determined by the InsulationScore method. We plotted $500 \mathrm{~kb}$ away from the diagonal and $500 \mathrm{~kb}$ on either side of the TAD boundary. To remove biases introduced by the contact matrix diagonal, we excluded the $30 \mathrm{~kb}$ closest to the diagonal.

Boxplots were generated using boundary strength scores determined by both InsulationScore and Hicratio methods. All TAD boundaries were plotted for the InsulationScore boxplots. For Hicratio boxplots, outliers were removed by filtering out TAD boundaries with a Hicratio score greater than twelve or less than one.

Western blot. At the appropriate timepoints, cells were trypsinized using standard protocols, and up to 5 million cells were collected by centrifugation (1000 rcf, $5 \mathrm{~min}$, room temperature). After carefully removing the media from the pellet, cells were resuspended in $300 \mathrm{ml}$ of RIPA buffer (Thermo Scientific, I89900), supplemented with protease inhibitors/EDTA (GenDepot 50-101-5485), and phosphatase inhibitors (GenDepot 50-101-5488). Cells were lysed by repeated pipetting followed by incubation, on ice, for $10 \mathrm{~min}$. DNA was degraded by adding $1.5 \mu \mathrm{l}$ of micrococcal nuclease (0.5 $\mathrm{U} \mathrm{ml}^{-1}$ stock, Thermo Scientific, FEREN0181) and $3 \mu \mathrm{l}$ of calcium chloride (100 $\mathrm{mM}$ stock) to each lysate, followed by incubation at $37^{\circ} \mathrm{C}$ for $15 \mathrm{~min}$. Nuclease was inactivated by subsequent incubations at $68^{\circ} \mathrm{C}$ for $15 \mathrm{~min}$, and ice for $10 \mathrm{~min}$. Cell debris was removed by centrifugation at $14,000 \mathrm{rcf}$ for $15 \mathrm{~min}$. Protein samples were stored at $-80^{\circ} \mathrm{C}$ until use. Protein concentration was measured using the BCA Protein Assay Kit. Thermo (234225), according to the manufacturer's instructions.

Denatured protein samples were resolved using a mini gel tank (Invitrogen, A25977) for $35 \mathrm{~min}$ at $200 \mathrm{~V}$, using $4-12 \%$ Bis-Tris Plus gels (Invitrogen, NW04120BOX) for all other targets. Protein was transferred to low fluorescence PVDF membranes using the mini Bolt Blotting System (Invitrogen, B1000), along with system-specific reagents. Blotting was performed using the Odyssey TBS blocking system (Licor, 927-400000), according to the manufacturer's protocol. Briefly, membranes were activated with methanol after transfer ( $1 \mathrm{~min}, \mathrm{RT})$, washed twice with milliQ water (5 min), twice with TBS ( $2 \mathrm{~min}$ ), and blocked with Odyssey TBS blocking solution for $1 \mathrm{~h}$ at RT. Primary antibodies were diluted in blocking buffer (containing $0.2 \%$ Tween), and incubated overnight at $4{ }^{\circ} \mathrm{C}$ as follows: $\gamma$-H2AX (1:1000, mouse monoclonal [9F3], Abcam). Beta actin was used as a loading control, using the appropriate antibody (1:10,000; rabbit polyclonal PA1-16889, Thermo Fisher or 1:10,000; mouse monoclonal MA1-140, Thermo Fisher) concomitantly with targets of interest. Detection was carried out using the following secondary antibodies: goat anti-rabbit (1:10,000; IRDye 680RD [92568071]; Licor) and goat anti-mouse (1:10,000, IRDye 800CW [95-32210]; Licor). Secondary antibodies were diluted in Odyssey TBS blocking buffer containing $0.2 \%$ Tween and $0.01 \%$ SDS. Membranes were incubated in secondary antibody for $1 \mathrm{~h}$ at RT, followed by three washes (TBST, $5 \mathrm{~min}$ ea.).

Images of near infrared fluorescent signal were acquired using an Odyssey scanner (Licor) in both the red and green channels. Signal was quantitated using the Image Studio software (Licor).

Reporting summary. Further information on research design is available in the Nature Research Reporting Summary linked to this article.

\section{Data availability}

All relevant data supporting the key findings of this study are available within the article and its Supplementary Information files or from the corresponding author on reasonable request. The Hi-C data generated in this study have been deposited in Gene Expression Omnibus (GEO) under accession number 'GSE136899'. Source data are provided with this paper. 


\section{Code availability}

The majority of software and code used for the analyses presented are available as previously published public github packages, as described in the Methods section. Custom scripts for calculating HiCRatio TAD boundaries and measuring Hi-C reproducibility are available from [https://github.com/rpmccordlab/XrayHiCAnalysis].

Received: 20 August 2019; Accepted: 21 October 2020;

Published online: 02 December 2020

\section{References}

1. Morgan, W. F., Day, J. P., Kaplan, M. I., McGhee, E. M. \& Limoli, C. L. Genomic instability induced by ionizing radiation. Radiat. Res. 146, 247-258 (1996).

2. Schwarzer, W. et al. Two independent modes of chromatin organization revealed by cohesin removal. Nature 551, 51 (2017).

3. Pope, B. D. et al. Topologically associating domains are stable units of replication-timing regulation. Nature 515, 402 (2014).

4. Naumova, N. et al. Organization of the mitotic chromosome. Science 342, 948-953 (2013).

5. Golloshi, R., Sanders, J. T. \& McCord, R. P. Genome organization during the cell cycle: unity in division. Wires Syst. Biol. Med. 9, e1389 (2017).

6. Balajee, A. S. et al. Investigation of spatial organization of chromosome territories in chromosome exchange aberrations after ionizing radiation exposure. Health Phys. 115, 77-89 (2018).

7. Zhang, Y. et al. Spatial organization of the mouse genome and its role in recurrent chromosomal translocations. Cell 148, 908-921 (2012).

8. Hnisz, D., Day, D. S. \& Young, R. A. Insulated neighborhoods: structural and functional units of mammalian gene control. Cell 167, 1188-1200 (2016).

9. Misteli, T. \& Soutoglou, E. The emerging role of nuclear architecture in DNA repair and genome maintenance. Nat. Rev. Mol. Cell Biol. 10, 243-254 (2009).

10. Clouaire, T. et al. Comprehensive mapping of histone modifications at DNA double-strand breaks deciphers repair pathway chromatin signatures. Mol. Cell 72, 250-262 e256 (2018).

11. Fortuny, A. \& Polo, S. E. The response to DNA damage in heterochromatin domains. Chromosoma 127, 291-300 (2018).

12. Jakob, B. et al. DNA double-strand breaks in heterochromatin elicit fast repair protein recruitment, histone $\mathrm{H} 2 \mathrm{AX}$ phosphorylation and relocation to euchromatin. Nucleic Acids Res. 39, 6489-6499 (2011).

13. Noon, A. T. et al. 53BP1-dependent robust localized KAP-1 phosphorylation is essential for heterochromatic DNA double-strand break repair. Nat. Cell Biol. 12, 177-184 (2010).

14. Natale, F. et al. Identification of the elementary structural units of the DNA damage response. Nat. Commun. 8, 15760 (2017).

15. Smith, M. J., Bryant, E. E. \& Rothstein, R. Increased chromosomal mobility after DNA damage is controlled by interactions between the recombination machinery and the checkpoint. Genes Dev. 32, 1242-1251 (2018).

16. Aymard, F. et al. Genome-wide mapping of long-range contacts unveils clustering of DNA double- strand breaks at damaged active genes. Nat. Struct. Mol. Biol. 24, 353-361 (2017).

17. Lang, F. et al. CTCF prevents genomic instability by promoting homologous recombination- directed DNA double-strand break repair. Proc. Natl Acad. Sci. USA 114, 10912-10917 (2017).

18. Hilmi, K. et al. CTCF facilitates DNA double-strand break repair by enhancing homologous recombination repair. Sci. Adv. 3, e1601898 (2017)

19. Han, D., Chen, Q., Shi, J., Zhang, F. \& Yu, X. CTCF participates in DNA damage response via poly(ADP- ribosyl)ation. Sci. Rep. 7, 43530 (2017).

20. Kim, J. S., Krasieva, T. B., LaMorte, V., Taylor, A. M. \& Yokomori, K. Specific recruitment of human cohesin to laser-induced DNA damage. J. Biol. Chem. 277, 45149-45153 (2002).

21. Alipour, E. \& Marko, J. F. Self-organization of domain structures by DNAloop-extruding enzymes. Nucleic Acids Res. 40, 11202-11212 (2012).

22. Goloborodko, A., Marko, J. F. \& Mirny, L. A. Chromosome compaction by active loop extrusion. Biophys. J. 110, 2162-2168 (2016).

23. Fudenberg, G. et al. Formation of chromosomal domains by loop extrusion. Cell Rep. 15, 2038-2049 (2016).

24. Rao, S. S. P. et al. Cohesin loss eliminates all loop domains. Cell 171, 305-320 e324 (2017).

25. Dixon, J. R. et al. Topological domains in mammalian genomes identified by analysis of chromatin interactions. Nature 485, 376-380 (2012).

26. Zuin, J. et al. Cohesin and CTCF differentially affect chromatin architecture and gene expression in human cells. Proc. Natl Acad. Sci. USA 111, 996-1001 (2014).

27. Lupianez, D. G. et al. Disruptions of topological chromatin domains cause pathogenic rewiring of gene-enhancer interactions. Cell 161, 1012-1025 (2015).

28. Hnisz, D. et al. Activation of proto-oncogenes by disruption of chromosome neighborhoods. Science 351, 1454-1458 (2016).
29. Arnould, C. et al. Loop extrusion as a mechanism for DNA Double-Strand Breaks repair foci formation. Preprint at https://www.biorxiv.org/content/ 10.1101/2020.02.12.945311v1 (2020)

30. Kesavan, P. S., Bohra, D., Roy, S. \& Mazumder, A. Monitoring global changes in chromatin compaction states upon localized DNA damage, with tools of fluorescence anisotropy. Mol. Biol. Cell 31, 1403-1410 (2020).

31. Lieberman-Aiden, E. et al. Comprehensive mapping of long-range interactions reveals folding principles of the human genome. Science 326, 289-293 (2009).

32. Bernhard, E. J., Maity, A., Muschel, R. J. \& McKenna, W. G. Effects of ionizing radiation on cell cycle progression. A review. Radiat. Environ. Biophys. 34, 79-83 (1995).

33. Pawlik, T. M. \& Keyomarsi, K. Role of cell cycle in mediating sensitivity to radiotherapy. Int. J. Radiat. Oncol. Biol. Phys. 59, 928-942 (2004).

34. Bouquet, F., Muller, C. \& Salles, B. The loss of gammaH2AX signal is a marker of DNA double strand breaks repair only at low levels of DNA damage. Cell Cycle 5, 1116-1122 (2006).

35. Kuo, L. J. \& Yang, L. X. Gamma-H2AX-a novel biomarker for DNA doublestrand breaks. Vivo 22, 305-309 (2008).

36. Iliakis, G. The role of DNA double strand breaks in lonizing radiation-induced killing of eukaryotic cells. Bioessays 13, 641-648 (1991).

37. Marti, T. M., Hefner, E., Feeney, L., Natale, V. \& Cleaver, J. E. H2AX phosphorylation within the G1 phase after UV irradiation depends on nucleotide excision repair and not DNA double-strand breaks. Proc. Natl Acad. Sci. USA 103, 9891-9896 (2006).

38. Rabl, C. Über zelltheilung. Morphologisches Jahrb. 10, 214-330 (1885).

39. Nora, E. P. et al. Targeted degradation of CTCF decouples local insulation of chromosome domains from genomic compartmentalization. Cell 169, 930-944 e922 (2017).

40. Franke, M. et al. Formation of new chromatin domains determines pathogenicity of genomic duplications. Nature 538, 265 (2016).

41. Crane, E. et al. Condensin-driven remodelling of X chromosome topology during dosage compensation. Nature 523, 240-U299 (2015).

42. Gong, Y. et al. Stratification of TAD boundaries reveals preferential insulation of super-enhancers by strong boundaries. Nat. Commun. 9, 542 (2018).

43. Rao, S. S. et al. A 3D map of the human genome at kilobase resolution reveals principles of chromatin looping. Cell 159, 1665-1680 (2014).

44. Hustedt, N. \& Durocher, D. The control of DNA repair by the cell cycle. Nat. Cell Biol. 19, 1-9 (2017).

45. Hickson, I. et al. Identification and characterization of a novel and specific inhibitor of the ataxia- telangiectasia mutated kinase ATM. Cancer Res. 64, 9152-9159 (2004)

46. Matsuoka, S. et al. Ataxia telangiectasia-mutated phosphorylates Chk2 in vivo and in vitro. Proc. Natl Acad. Sci. USA 97, 10389-10394 (2000).

47. Shiloh, Y. Ataxia-telangiectasia and the Nijmegen breakage syndrome: Related disorders but genes apart. Annu Rev. Genet. 31, 635-662 (1997).

48. Friedenson, B. The BRCA1/2 pathway prevents hematologic cancers in addition to breast and ovarian cancers. BMC Cancer 7, 152 (2007).

49. Friesner, J. D., Liu, B., Culligan, K. \& Britt, A. B. Ionizing radiation-dependent gamma-H2AX focus formation requires ataxia telangiectasia mutated and ataxia telangiectasia mutated and Rad3- related. Mol. Biol. Cell 16, 2566-2576 (2005).

50. Aymard, F. \& Legube, G. A. TAD closer to ATM. Mol. Cell Oncol. 3, e1134411 (2016).

51. Clouaire, T., Marnef, A. \& Legube, G. Taming tricky DSBs: ATM on duty. DNA Repair (Amst.) 56, 84-91 (2017).

52. Bakr, A. et al. Involvement of ATM in homologous recombination after end resection and RAD51 nucleofilament formation. Nucleic Acids Res. 43, 3154-3166 (2015)

53. Canela, A. et al. Genome organization drives chromosome fragility. Cell 170, 507 (2017).

54. Amat, R. et al. Rapid reversible changes in compartments and local chromatin organization revealed by hyperosmotic shock. Genome Res. 29, 18-28 (2019).

55. Ray, J. et al. Chromatin conformation remains stable upon extensive transcriptional changes driven by heat shock. Proc. Natl Acad. Sci. USA 116, 19431-19439 (2019).

56. Habash, M. et al. Clinical and functional assays of radiosensitivity and radiation-induced second cancer. Cancers (Basel) 9, 147 (2017).

57. Deschavanne, P. J. \& Fertil, B. A review of human cell radiosensitivity in vitro. Int. J. Radiat. Oncol. Biol. Phys. 34, 251-266 (1996).

58. Schmitt, A. D. et al. A compendium of chromatin contact maps reveals spatially active regions in the human genome. Cell Rep. 17, 2042-2059 (2016).

59. Luo, H. et al. Regulation of intra-S phase checkpoint by ionizing radiation (IR)-dependent and IR- independent phosphorylation of SMC3. J. Biol. Chem. 283, 19176-19183 (2008)

60. Grawunder, U. et al. Activity of DNA ligase IV stimulated by complex formation with XRCC4 protein in mammalian cells. Nature 388, 492-495 (1997).

61. Wilson, T. E., Grawunder, U. \& Lieber, M. R. Yeast DNA ligase IV mediates non-homologous DNA end joining. Nature 388, 495-498 (1997). 
62. Ahnesorg, P., Smith, P. \& Jackson, S. P. XLF interacts with the XRCC4-DNA ligase IV complex to promote DNA nonhomologous end-joining. Cell 124, 301-313 (2006).

63. Shiotani, B. \& Zou, L. Single-stranded DNA orchestrates an ATM-to-ATR switch at DNA breaks. Mol. Cell 33, 547-558 (2009).

64. Sultana, R. et al. Ataxia telangiectasia mutated and Rad3 related (ATR) protein kinase inhibition is synthetically lethal in XRCC1 deficient ovarian cancer cells. PLOS ONE 8, e57098 (2013).

65. Lin, Y. et al. APE2 promotes DNA damage response pathway from a singlestrand break. Nucleic Acids Res. 46, 2479-2494 (2018).

66. Golloshi, R., Sanders, J. T. \& McCord, R. P. Iteratively improving Hi-C experiments one step at a time. Methods 142, 47-58 (2018).

67. Imakaev, $\mathrm{M}$. et al. Iterative correction of $\mathrm{Hi}-\mathrm{C}$ data reveals hallmarks of chromosome organization. Nat. Methods 9, 999-1003 (2012).

68. Ursu, O. et al. GenomeDISCO: a concordance score for chromosome conformation capture experiments using random walks on contact map graphs. Bioinformatics 34, 2701-2707 (2018).

\section{Acknowledgements}

We thank Dianne Trent and Stephen Kania in the Biomedical and Diagnostic Sciences department of the University of Tennessee Veterinary Medical Center for advice and assistance with flow cytometry and Mariano Labrador for helpful discussions. This work was supported in part by a grant from Oak Ridge Associated Universities Directed Research and Development (ODRD) to A.B. and R.P.M. and by NIH NIGMS grant R35GM133557 to R.P.M.

\section{Author contributions}

R.P.M., A.B., and J.T.S. devised the project. Hi-C experiments were performed by J.T.S., T.F., and R.G. Image quantification and analysis was performed by M.S., A.H., R.S.M., and J.T.S. Western blot preparation and analysis was performed by R.S.M. and J.T.S. Hi-C analysis was performed by J.T.S., T.F., Y.X., and R.P.M. The manuscript was prepared by J.T.S., T.F., and R.P.M., with input from A.B., R.S.M., and R.G.

\section{Competing interests}

The authors declare no competing interests.

\section{Additional information}

Supplementary information is available for this paper at https://doi.org/10.1038/s41467020-20047-w.

Correspondence and requests for materials should be addressed to R.P.M.

Peer review information Nature Communications thanks Michael Witcher and the other, anonymous, reviewer(s) for their contribution to the peer review of this work.

Reprints and permission information is available at http://www.nature.com/reprints

Publisher's note Springer Nature remains neutral with regard to jurisdictional claims in published maps and institutional affiliations.

(c) (i) Open Access This article is licensed under a Creative Commons Attribution 4.0 International License, which permits use, sharing, adaptation, distribution and reproduction in any medium or format, as long as you give appropriate credit to the original author(s) and the source, provide a link to the Creative Commons license, and indicate if changes were made. The images or other third party material in this article are included in the article's Creative Commons license, unless indicated otherwise in a credit line to the material. If material is not included in the article's Creative Commons license and your intended use is not permitted by statutory regulation or exceeds the permitted use, you will need to obtain permission directly from the copyright holder. To view a copy of this license, visit http://creativecommons.org/ licenses/by/4.0/.

(C) The Author(s) 2020 